\title{
Compact exact Lagrangian intersections in cotangent bundles via sheaf quantization
}

\author{
Yuichi Ike
}

October 29, 2019

\begin{abstract}
We show that the cardinality of the transverse intersection of two compact exact Lagrangian submanifolds in a cotangent bundle is bounded from below by the dimension of the Hom space of sheaf quantizations of the Lagrangians in Tamarkin's category. Our sheaf-theoretic method can also deal with clean and degenerate Lagrangian intersections.
\end{abstract}

\section{Introduction}

The study of Lagrangian intersections, especially intersections of exact Lagrangian submanifolds in cotangent bundles is an important problem in symplectic geometry. In this paper, we study them using a method based on microlocal sheaf theory, more precisely, Tamarkin's category and Guillermou's sheaf quantization. We state our main result and its corollary in Subsection 1.2.

\subsection{Applications of microlocal sheaf theory to symplectic geometry}

Microlocal sheaf theory was introduced and systematically developed by Kashiwara and Schapira [KS90]. One of the key ingredients of the theory is the notion of microsupports of sheaves. In the sequel, let $\mathbf{k}$ be a field. Moreover, let $X$ be a $C^{\infty}$-manifold and denote by $\mathbf{D}^{\mathrm{b}}(X)$ the bounded derived category of sheaves of $\mathbf{k}$-vector spaces. For an object $F \in \mathbf{D}^{\mathrm{b}}(X)$, its microsupport $\mathrm{SS}(F)$ is defined as the set of directions in which the cohomology of $F$ cannot be extended isomorphically. The microsupport is a closed subset of the cotangent bundle $T^{*} X$ and conic, that is, invariant under the action of $\mathbb{R}_{>0}$ on $T^{*} X$.

Tamarkin [Tam18] proposed a new approach to symplectic geometry, which is based on microlocal sheaf theory. A sheaf whose microsupport coincides with a given conic Lagrangian submanifold of a cotangent bundle (outside the zero-section) is called a sheaf quantization of the Lagrangian. For a non-conic Lagrangian, one can consider a sheaf quantization by adding a variable and "conifying" it. Using sheaf quantizations, Tamarkin studied the intersections of particular Lagrangian submanifolds. After his work, GuillermouKashiwara-Schapira GKS12 and Guillermou Gui12, Gui16 proved the existence of sheaf quantizations of graphs of Hamiltonian isotopies and compact exact Lagrangian submanifolds in cotangent bundles, respectively. See Section 3 for more details. Note that sheaftheoretic approaches to symplectic geometry also appeared in [KO01, NZ09, Nad09]. 


\subsection{Our results}

In this paper, we prove that the cardinality of the transverse intersection of compact exact Lagrangian submanifolds in a cotangent bundle is bounded from below by the dimension of the Hom space of sheaf quantizations of the Lagrangians in Tamarkin's category. More generally, provided $\mathbf{k}=\mathbb{F}_{2}=\mathbb{Z} / 2 \mathbb{Z}$, we show that a clean version of the estimate holds with "cardinality" replaced by "total $\mathbb{F}_{2}$-Betti number".

In what follows, let $M$ be a compact connected $C^{\infty}$-manifold without boundary and denote by $T^{*} M$ its cotangent bundle. We also denote by $(x ; \xi)$ a local homogeneous coordinate system. We regard $T^{*} M$ as an exact symplectic manifold equipped with the Liouville 1-form $\alpha=\langle\xi, d x\rangle$. A submanifold $L$ of dimension $\operatorname{dim} M$ in $T^{*} M$ is said to be exact Lagrangian if $\left.\alpha\right|_{L}$ is exact. The main result of this paper is the following. See Section 3 for the definitions of simple sheaf quantizations, $\mathcal{H o m}^{\star}$, and Tamarkin's category $\mathcal{T}(M)$.

Theorem 1.1 (see Theorem 4.17). For $i=1,2$, let $L_{i}$ be a compact connected exact Lagrangian submanifold and $F_{i} \in \mathbf{D}^{\mathrm{b}}(M \times \mathbb{R})$ be a simple sheaf quantization associated with $L_{i}$ and a function $f_{i}: L_{i} \rightarrow \mathbb{R}$ satisfying $d f_{i}=\left.\alpha\right|_{L_{i}}$. Assume that $L_{1}$ and $L_{2}$ intersect cleanly, that is, $L_{1} \cap L_{2}$ is a submanifold of $T^{*} M$ and $T_{p}\left(L_{1} \cap L_{2}\right)=T_{p} L_{1} \cap T_{p} L_{2}$ for any $p \in L_{1} \cap L_{2}$. Let $L_{1} \cap L_{2}=\bigsqcup_{j=1}^{n} C_{j}$ be the decomposition into connected components and define $f_{21}\left(C_{j}\right):=f_{2}(p)-f_{1}(p)$ for some $p \in C_{j}$ (independent of the choice of $p$ ). Moreover, let $a, b \in \mathbb{R}$ with $a<b$ or $a \in \mathbb{R}, b=+\infty$. Then, for $\mathbf{k}=\mathbb{F}_{2}=\mathbb{Z} / 2 \mathbb{Z}$, one has

$$
\begin{aligned}
& \sum_{a \leq f_{21}\left(C_{j}\right)<b} \sum_{k \in \mathbb{Z}} \operatorname{dim}_{\mathbb{F}_{2}} H^{k}\left(C_{j} ; \mathbb{F}_{2}\right) \\
& \quad \geq \sum_{k \in \mathbb{Z}} \operatorname{dim}_{\mathbb{F}_{2}} H^{k} R \Gamma_{M \times[a, b)}\left((-\infty, b) ; \mathcal{H o m}^{\star}\left(F_{2}, F_{1}\right)\right) .
\end{aligned}
$$

In particular,

$$
\sum_{j=1}^{n} \sum_{k \in \mathbb{Z}} \operatorname{dim}_{\mathbb{F}_{2}} H^{k}\left(C_{j} ; \mathbb{F}_{2}\right) \geq \sum_{k \in \mathbb{Z}} \operatorname{dim}_{\mathbb{F}_{2}} \operatorname{Hom}_{\mathcal{T}(M)}\left(F_{2}, F_{1}[k]\right) .
$$

If $L_{1}$ and $L_{2}$ intersect transversally, the inequalities hold for any field $\mathbf{k}$, not only for $\mathbb{F}_{2}$.

We also have

$$
\operatorname{Hom}_{\mathcal{T}(M)}\left(F_{2}, F_{1}[k]\right) \simeq H^{k}(M ; \mathcal{L}) \quad \text { for any } k \in \mathbb{Z},
$$

where $\mathcal{L}$ is the locally constant sheaf of rank 1 on $M$ associated with $F_{1}$ and $F_{2}$ (see Proposition 4.2 for details). Combining this with Theorem 1.1, we obtain a purely sheaftheoretic proof of the following result of Nadler [Nad09] and Fukaya-Seidel-Smith [FSS08], as a corollary.

Corollary 1.2 ([Nad09, Thm. 1.3.1] and [FSS08, Thm. 1]). Let $L_{1}$ and $L_{2}$ be compact connected exact Lagrangian submanifolds of $T^{*} M$ intersecting transversally. Then

$$
\#\left(L_{1} \cap L_{2}\right) \geq \sum_{k \in \mathbb{Z}} \operatorname{dim} H^{k}(M ; \mathcal{L})
$$

for any rank 1 locally constant sheaf $\mathcal{L}$ on $M$ over any field $\mathbf{k}$. In particular, $\#\left(L_{1} \cap L_{2}\right) \geq$ $\sum_{k \in \mathbb{Z}} \operatorname{dim} H^{k}(M ; \mathbf{k})$. 
The proof of Theorem 1.1 goes as follows. First, we apply the Morse-Bott inequality for sheaves (see Theorem 2.10) to $H:=\mathcal{H o m}^{\star}\left(F_{2}, F_{1}\right)$ and the function $M \times \mathbb{R} \rightarrow \mathbb{R},(x, t) \mapsto t$, and obtain

$$
\begin{gathered}
\sum_{a \leq c<b} \sum_{k \in \mathbb{Z}} \operatorname{dim} H^{k} R \Gamma\left(M \times\{c\} ;\left.R \Gamma_{M \times[c,+\infty)}(H)\right|_{M \times\{c\}}\right) \\
\geq \sum_{k \in \mathbb{Z}} \operatorname{dim} H^{k} R \Gamma_{M \times[a, b)}(M \times(-\infty, b) ; H) .
\end{gathered}
$$

In order to calculate the left-hand side of (1.5), we use the functor $\mu$ hom: $\mathbf{D}^{\mathrm{b}}(X)^{\mathrm{op}} \times$ $\mathbf{D}^{\mathrm{b}}(X) \rightarrow \mathbf{D}^{\mathrm{b}}\left(T^{*} X\right)$ introduced by Kashiwara-Schapira [KS90. Using the functor, we show the isomorphism

$$
R \Gamma\left(M \times\{c\} ;\left.R \Gamma_{M \times[c,+\infty)}(H)\right|_{M \times\{c\}}\right) \simeq R \Gamma\left(\Omega_{+} ;\left.\mu h o m\left(T_{c *} F_{2}, F_{1}\right)\right|_{\Omega_{+}}\right),
$$

where $T_{c}: M \times \mathbb{R} \rightarrow M \times \mathbb{R},(x, t) \mapsto(x, t+c)$ and $\Omega_{+}:=\{\tau>0\} \subset T^{*}(M \times \mathbb{R})$ with $(t ; \tau)$ being the homogeneous symplectic coordinate on $T^{*} \mathbb{R}$. The object $\left.\mu h o m\left(T_{c *} F_{2}, F_{1}\right)\right|_{\Omega_{+}}$ is supported in $\left\{(x, t ; \tau \xi, \tau) \mid \tau>0,(x ; \xi) \in L_{1} \cap L_{2}, t=f_{2}(x ; \xi)-f_{1}(x ; \xi)=c\right\}$ and isomorphic to a shift of the constant sheaf of rank 1 on the support. This completes the proof.

Remark 1.3. Even if the intersection is degenerate, (1.5) and (1.6) still hold, but the object $\left.\operatorname{\mu hom}\left(T_{c *} F_{2}, F_{1}\right)\right|_{\Omega_{+}}$is not necessarily locally constant on the support. In this sense, the family of sheaves $\left\{\left.\mu h o m\left(T_{c *} F_{2}, F_{1}\right)\right|_{\Omega_{+}}\right\}_{c}$ encodes the "contribution" from each possibly degenerate component of the intersection $L_{1} \cap L_{2}$. We will also explore the contribution in degenerate cases in Section A.

\subsection{Relation to Lagrangian intersection Floer theory}

Although our approach is purely sheaf-theoretic, it seems to be closely related to Floer cohomology and Fukaya categories. We briefly remark on the relation below. Tamarkin's category $\mathcal{T}(M)$ has the following properties:

(i) Hamiltonian invariance ([Tam18, GS14]),

(ii) the dimension of the cohomology of the clean intersection of two compact exact Lagrangian submanifolds is bounded from below by the dimension of the Hom space of simple sheaf quantizations (Theorem 1.1).

Moreover, as pointed out by T. Kuwagaki, the following also holds in $\mathcal{T}(M)$ :

(iii) a simple sheaf quantization associated with any compact connected exact Lagrangian submanifold is isomorphic to a simple sheaf quantization associated with the zerosection of $T^{*} M$ (see Proposition 4.4).

The Floer cohomology $\operatorname{HF}^{*}\left(L_{2}, L_{1}\right)$ has similar properties to (i) and (ii), though the approach is totally different. Floer cohomology for clean Lagrangian intersections was studied by Poźniak [Poź99], Frauenfelder [Fra04, Fukaya-Oh-Ohta-Ono [FOOO09a, FOOO09b, and Schmäschke [Sch16]. Moreover, Nadler [Nad09] and Fukaya-Seidel-Smith [FSS08, FSS09] proved the following, which corresponds to (iii): in the infinitesimal Fukaya category of $T^{*} M$, any relative spin compact connected exact Lagrangian submanifold of $T^{*} M$ with vanishing Maslov class is isomorphic to a shift of the zero-section. Note that their assumptions of relative spin and vanishing Maslov class can be removed, thanks to results 
of Abouzaid Abo12, and Abouzaid-Kragh [Kra13, respectively. We also remark that Guillermou Gui12, Gui16 gave a sheaf-theoretic proof for the relative spin property and the vanishing of the Maslov class.

During the preparation of this paper, C. Viterbo announced 11 that he had found some relation between $\mathcal{H o m}^{\star}\left(F_{2}, F_{1}\right)$ and the Floer cochain complex $\operatorname{CF}\left(L_{2}, L_{1}\right)$.

\subsection{Outline of this paper}

This paper is organized as follows. In Section 2, we recall the microlocal sheaf theory due to Kashiwara and Schapira [KS90]. In Section 3 we review the results of [Tam18, GKS12, GS14, Gui12, Gui16 on Tamarkin's non-displaceability theorem, and sheaf quantization of Hamiltonian isotopies and compact exact Lagrangian submanifolds in cotangent bundles. In Section 4 we prove the isomorphism (1.3) and Theorem 1.1. In Section A we briefly remark that our method can deal with degenerate Lagrangian intersections, using very simple examples. In Section B we prove the "functoriality" of simple sheaf quantizations with respect to Hamiltonian diffeomorphisms. In Section C by Tomohiro Asano, we relate the shift of a simple sheaf quantization of a Lagrangian to the grading in Lagrangian intersection Floer cohomology theory.

\section{Acknowledgments}

The author wishes to express his sincere gratitude to Stéphane Guillermou for many helpful discussions. He is also very grateful to Pierre Schapira for many enlightening discussions and helpful advice and to Tomohiro Asano for many fruitful discussions and kindly writing Section C. The author also thanks Vincent Humilière and Tatsuki Kuwagaki for many stimulating discussions. He expresses his gratitude to IMJ-PRG and "equipe Analyse Algébrique" for hospitality during the preparation of this paper. This work was supported by a Grant-in-Aid for JSPS Fellows 15 J07993 and the Program for Leading Graduate Schools, MEXT, Japan.

\section{Preliminaries on microlocal sheaf theory}

In this paper, all manifolds are assumed to be real manifolds of class $C^{\infty}$ without boundary. Throughout this paper, let $\mathbf{k}$ be a field.

In this section we recall some definitions and results from [KS90]. We mainly follow the notation in KS90. Until the end of this section, let $X$ be a $C^{\infty}$-manifold without boundary.

\subsection{Geometric notions ([KS90, §4.3, §A.2])}

For a locally closed subset $A$ of $X$, we denote by $\bar{A}$ its closure and by $\operatorname{Int}(A)$ its interior. We also denote by $\Delta_{X}$ or simply $\Delta$ the diagonal of $X \times X$. We denote by $\tau_{X}: T X \rightarrow X$ the tangent bundle of $X$, and by $\pi_{X}: T^{*} X \rightarrow X$ the cotangent bundle of $X$. If there is no risk of confusion, we simply write $\tau$ and $\pi$ instead of $\tau_{X}$ and $\pi_{X}$, respectively. For a submanifold $M$ of $X$, one denotes by $T_{M} X$ the normal bundle to $M$ in $X$, and by $T_{M}^{*} X$ the conormal bundle to $M$ in $X$. In particular, $T_{X}^{*} X$ denotes the zero-section of $T^{*} X$. We set $\stackrel{\circ}{*}^{*} X:=T^{*} X \backslash T_{X}^{*} X$. For two subsets $S_{1}$ and $S_{2}$ of $X$, we denote by $C\left(S_{1}, S_{2}\right) \subset T X$ the normal cone of the pair $\left(S_{1}, S_{2}\right)$.

\footnotetext{
${ }^{1}$ In a seminar at IMJ-PRG on 10 October 2016.
} 
Let $f: X \rightarrow Y$ be a morphism of manifolds. With $f$ we associate the following morphisms and commutative diagram:

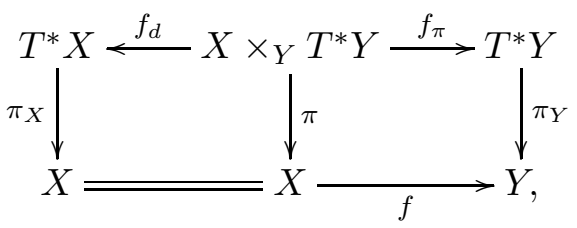

where $f_{\pi}$ is the projection and $f_{d}$ is induced by the transpose of the tangent map $f^{\prime}: T X \rightarrow$ $X \times_{Y} T Y$.

We denote by $(x ; \xi)$ a local homogeneous coordinate system on $T^{*} X$. The cotangent bundle $T^{*} X$ is an exact symplectic manifold with the Liouville 1 -form $\alpha=\langle\xi, d x\rangle$. We denote by $a: T^{*} X \rightarrow T^{*} X,(x ; \xi) \mapsto(x ;-\xi)$ the antipodal map. For a subset $A$ of $T^{*} X$, we denote by $A^{a}$ its image under the map $a$. We also denote by $\mathbf{h}: T^{*} T^{*} X \stackrel{\sim}{\rightarrow} T T^{*} X$ the Hamiltonian isomorphism given in local coordinates by $\mathbf{h}\left(d x_{i}\right)=-\partial / \partial \xi_{i}$ and $\mathbf{h}\left(d \xi_{i}\right)=$ $\partial / \partial x_{i}$.

\subsection{Microsupports of sheaves ([KS90, §5.1, §5.4, §6.1])}

We denote by $\mathbf{k}_{X}$ the constant sheaf with stalk $\mathbf{k}$ and by $\operatorname{Mod}\left(\mathbf{k}_{X}\right)$ the abelian category of sheaves of $\mathbf{k}$-vector spaces on $X$. Moreover, we denote by $\mathbf{D}^{\mathrm{b}}(X)=\mathbf{D}^{\mathrm{b}}\left(\operatorname{Mod}\left(\mathbf{k}_{X}\right)\right)$ the bounded derived category of $\operatorname{Mod}\left(\mathbf{k}_{X}\right)$. One can define Grothendieck's six operations between derived categories of sheaves $R \mathcal{H}$ om, $\otimes, R f_{*}, f^{-1}, R f_{!}, f^{!}$for a morphism of manifolds $f: X \rightarrow Y$. Since we work over the field $\mathbf{k}$, we simply write $\otimes$ instead of $\stackrel{L}{\otimes}$. Moreover, for $F \in \mathbf{D}^{\mathrm{b}}(X)$ and $G \in \mathbf{D}^{\mathrm{b}}(Y)$, we define their external tensor product $F \otimes G \in \mathbf{D}^{\mathrm{b}}(X \times Y)$ by $F \otimes G:=q_{X}^{-1} F \otimes q_{Y}^{-1} G$, where $q_{X}: X \times Y \rightarrow X$ and $q_{Y}: X \times Y \rightarrow Y$ are the projections. For a locally closed subset $Z$ of $X$, we denote by $\mathbf{k}_{Z}$ the zero-extension of the constant sheaf with stalk $\mathbf{k}$ on $Z$ to $X$, extended by 0 on $X \backslash Z$. Moreover, for a locally closed subset $Z$ of $X$ and $F \in \mathbf{D}^{\mathrm{b}}(X)$, we define $F_{Z}, R \Gamma_{Z}(F) \in \mathbf{D}^{\mathrm{b}}(X)$ by

$$
F_{Z}:=F \otimes \mathbf{k}_{Z}, \quad R \Gamma_{Z}(F):=R \mathcal{H o m}\left(\mathbf{k}_{Z}, F\right) .
$$

One denotes by $\omega_{X} \in \mathbf{D}^{\mathrm{b}}(X)$ the dualizing complex on $X$, that is, $\omega_{X}:=a_{X}^{!} \mathbf{k}$, where $a_{X}: X \rightarrow$ pt is the natural morphism. Note that $\omega_{X}$ is isomorphic to $\operatorname{or}_{X}[\operatorname{dim} X]$, where or $_{X}$ is the orientation sheaf on $X$. More generally, for a morphism of manifolds $f: X \rightarrow Y$, we denote by $\omega_{f}=\omega_{X / Y}:=f^{!} \mathbf{k}_{Y} \simeq \omega_{X} \otimes f^{-1} \omega_{Y}^{\otimes-1}$ the relative dualizing complex.

Let us recall the definition of the microsupport $\operatorname{SS}(F)$ of $F \in \mathbf{D}^{\mathrm{b}}(X)$.

Definition 2.1 ([KS90, Def. 5.1.2]). Let $F \in \mathbf{D}^{\mathrm{b}}(X)$ and $p \in T^{*} X$. One says that $p \notin \mathrm{SS}(F)$ if there is a neighborhood $U$ of $p$ in $T^{*} X$ such that for any $x_{0} \in X$ and any $C^{\infty}$-function $\varphi$ on $X$ (defined on a neighborhood of $x_{0}$ ) satisfying $d \varphi\left(x_{0}\right) \in U$, one has $R \Gamma_{\left\{\varphi \geq \varphi\left(x_{0}\right)\right\}}(F)_{x_{0}} \simeq 0$.

One can check the following properties:

(i) The microsupport of an object in $\mathbf{D}^{\mathrm{b}}(X)$ is a conic (i.e., invariant under the action of $\mathbb{R}_{>0}$ on $T^{*} X$ ) closed subset of $T^{*} X$.

(ii) For an object $F \in \mathbf{D}^{\mathrm{b}}(X)$, one has $\operatorname{SS}(F) \cap T_{X}^{*} X=\pi(\operatorname{SS}(F))=\operatorname{Supp}(F)$.

(iii) The microsupports satisfy the triangle inequality: if $F_{1} \rightarrow F_{2} \rightarrow F_{3} \stackrel{+1}{\rightarrow}$ is a distinguished triangle in $\mathbf{D}^{\mathrm{b}}(X), \operatorname{SS}\left(F_{i}\right) \subset \mathrm{SS}\left(F_{j}\right) \cup \operatorname{SS}\left(F_{k}\right)$ for $j \neq k$. 
We also use the notation $\mathrm{SS}^{\circ}(F):=\mathrm{SS}(F) \cap \stackrel{\circ}{T}^{*} X=\mathrm{SS}(F) \backslash T_{X}^{*} X$.

We denote by $\mathbf{D}(X)=\mathbf{D}\left(\operatorname{Mod}\left(\mathbf{k}_{X}\right)\right)$ the (unbounded) derived category of sheaves of k-vector spaces on $X$. An object $F \in \mathbf{D}(X)$ is said to be locally bounded if for any relatively compact open subset $U$ of $X$, one has $\left.F\right|_{U} \in \mathbf{D}^{\mathrm{b}}(U)$. We denote by $\mathbf{D}^{\mathrm{lb}}(X)$ the full subcategory of $\mathbf{D}(X)$ consisting of locally bounded objects. The microsupport of an object in $\mathbf{D}^{\mathrm{lb}}(X)$ can be defined in totally the same way as in Definition 2.1, since it is a local notion.

Example 2.2. (i) If $F$ is a non-zero locally constant sheaf on a connected manifold $X$, then $\operatorname{SS}(F)=T_{X}^{*} X$. Conversely, if $\operatorname{SS}(F) \subset T_{X}^{*} X$ then the cohomology sheaves $H^{k}(F)$ are locally constant for all $k \in \mathbb{Z}$.

(ii) Let $M$ be a closed submanifold of $X$. Then $\operatorname{SS}\left(\mathbf{k}_{M}\right)=T_{M}^{*} X \subset T^{*} X$.

(iii) Let $\varphi: X \rightarrow \mathbb{R}$ be a $C^{\infty}$-function and assume that $d \varphi(x) \neq 0$ for any $x \in \varphi^{-1}(0)$. Set $U:=\{x \in X \mid \varphi(x)>0\}$ and $Z:=\{x \in X \mid \varphi(x) \geq 0\}$. Then

$$
\begin{aligned}
& \mathrm{SS}\left(\mathbf{k}_{U}\right)=\left.T_{X}^{*} X\right|_{U} \cup\{(x ; \lambda d \varphi(x)) \mid \varphi(x)=0, \lambda \leq 0\}, \\
& \mathrm{SS}\left(\mathbf{k}_{Z}\right)=\left.T_{X}^{*} X\right|_{Z} \cup\{(x ; \lambda d \varphi(x)) \mid \varphi(x)=0, \lambda \geq 0\} .
\end{aligned}
$$

The following proposition is called (a particular case of) the microlocal Morse lemma. See [KS90, Prop. 5.4.17 and Cor. 5.4.19] for more details. The classical theory corresponds to the case $F$ is the constant sheaf $\mathbf{k}_{X}$.

Proposition 2.3. Let $F \in \mathbf{D}^{\mathrm{b}}(X)$ and $\varphi: X \rightarrow \mathbb{R}$ be a $C^{\infty}$-function. Moreover, let $a, b \in \mathbb{R}$ with $a<b$ or $a \in \mathbb{R}, b=+\infty$. Assume that

(1) $\varphi$ is proper on $\operatorname{Supp}(F)$,

(2) $d \varphi(x) \notin \mathrm{SS}(F)$ for any $x \in \varphi^{-1}([a, b))$.

Then the canonical morphism

$$
R \Gamma\left(\varphi^{-1}((-\infty, b)) ; F\right) \longrightarrow R \Gamma\left(\varphi^{-1}((-\infty, a)) ; F\right)
$$

is an isomorphism.

By using microsupports, we can microlocalize the category $\mathbf{D}^{\mathrm{b}}(X)$. Let $A \subset T^{*} X$ be a subset and set $\Omega=T^{*} X \backslash A$. We denote by $\mathbf{D}_{A}^{\mathrm{b}}(X)$ the subcategory of $\mathbf{D}^{\mathrm{b}}(X)$ consisting of sheaves whose microsupports are contained in $A$. By the triangle inequality, the subcategory $\mathbf{D}_{A}^{\mathrm{b}}(X)$ is a triangulated subcategory. We define $\mathbf{D}^{\mathrm{b}}(X ; \Omega)$ as the localization of $\mathbf{D}^{\mathrm{b}}(X)$ by $\mathbf{D}_{A}^{\mathrm{b}}(X): \mathbf{D}^{\mathrm{b}}(X ; \Omega):=\mathbf{D}^{\mathrm{b}}(X) / \mathbf{D}_{A}^{\mathrm{b}}(X)$. A morphism $u: F \rightarrow G$ in $\mathbf{D}^{\mathrm{b}}(X)$ becomes an isomorphism in $\mathbf{D}^{\mathrm{b}}(X ; \Omega)$ if $u$ is embedded in a distinguished triangle $F \stackrel{u}{\rightarrow} G \rightarrow H \stackrel{+1}{\rightarrow}$ with $\mathrm{SS}(H) \cap \Omega=\emptyset$. For a closed subset $B$ of $\Omega, \mathbf{D}_{B}^{\mathrm{b}}(X ; \Omega)$ denotes the full triangulated subcategory of $\mathbf{D}^{\mathrm{b}}(X ; \Omega)$ consisting of $F$ with $\operatorname{SS}(F) \cap \Omega \subset B$. In the case $\Omega=\{p\}$ with $p \in T^{*} X$, we simply write $\mathbf{D}^{\mathrm{b}}(X ; p)$ instead of $\mathbf{D}^{\mathrm{b}}(X ;\{p\})$. Note that our notation is the same as in [KS90] and slightly differs from that of [Gui12, Gui16].

\subsection{Functorial operations ([KS90, §5.4])}

We consider bounds for the microsupports of proper direct images, non-characteristic inverse images, and RHom.

Definition 2.4 ([KS90, Def. 5.4.12]). Let $f: X \rightarrow Y$ be a morphism of manifolds and $A$ be a closed conic subset of $T^{*} Y$. The morphism $f$ is said to be non-characteristic for $A$ if

$$
f_{\pi}^{-1}(A) \cap f_{d}^{-1}\left(T_{X}^{*} X\right) \subset X \times_{Y} T_{Y}^{*} Y .
$$


See (2.1) for the notation $f_{\pi}$ and $f_{d}$. In particular, any submersion from $X$ to $Y$ is non-characteristic for any closed conic subset of $T^{*} Y$. Note that submersions are called smooth morphisms in [KS90]. One can show that if $f: X \rightarrow Y$ is non-characteristic for a closed conic subset $A$ of $T^{*} Y$, then $f_{d} f_{\pi}^{-1}(A)$ is a closed conic subset of $T^{*} X$.

Theorem 2.5 ([KS90, Prop. 5.4.4 and Prop. 5.4.13]). Let $f: X \rightarrow Y$ be a morphism of manifolds, $F \in \mathbf{D}^{\mathrm{b}}(X)$, and $G \in \mathbf{D}^{\mathrm{b}}(Y)$.

(i) Assume that $f$ is proper on $\operatorname{Supp}(F)$. Then $\operatorname{SS}\left(R f_{*} F\right) \subset f_{\pi} f_{d}^{-1}(\operatorname{SS}(F))$.

(ii) Assume that $f$ is non-characteristic for $\operatorname{SS}(G)$. Then the canonical morphism $f^{-1} G \otimes \omega_{f} \rightarrow f^{!} G$ is an isomorphism and $\operatorname{SS}\left(f^{-1} G\right) \cup \operatorname{SS}\left(f^{!} G\right) \subset f_{d} f_{\pi}^{-1}(\operatorname{SS}(G))$.

Proposition 2.6 ([KS90, Prop. 5.4.2]). For $i=1,2$, let $X_{i}$ be a manifold and denote by $q_{i}$ the projection $X_{1} \times X_{2} \rightarrow X_{i}$. Moreover, let $F_{i} \in \mathbf{D}^{\mathrm{b}}\left(X_{i}\right)$ for $i=1,2$. Then

$$
\operatorname{SS}\left(R \mathcal{H} \text { om }\left(q_{2}^{-1} F_{2}, q_{1}^{-1} F_{1}\right)\right) \subset \operatorname{SS}\left(F_{1}\right) \times \operatorname{SS}\left(F_{2}\right)^{a} .
$$

Using Proposition 2.6 and Theorem 2.5 (ii) for the diagonal embedding $\delta: X \rightarrow X \times X$, one can prove the following:

Proposition 2.7 ([KS90, Prop. 5.4.14 (ii)]). Let $F, G \in \mathbf{D}^{\mathrm{b}}(X)$ and assume that $\mathrm{SS}(F) \cap$ $\mathrm{SS}(G) \subset T_{X}^{*} X$. Then

$$
\operatorname{SS}(R \mathcal{H} o m(F, G)) \subset \mathrm{SS}(F)^{a}+\operatorname{SS}(G)
$$

where + is the fiberwise sum.

\subsection{Non-proper direct images ([Tam18, GS14])}

We consider estimates of the microsupports of non-proper direct images in special cases. Let $V_{1}$ and $V_{2}$ be finite-dimensional real vector spaces and consider a constant linear map $u: X \times V_{1} \rightarrow X \times V_{2}$. That is, we assume that there exists a linear map $u_{V}: V_{1} \rightarrow V_{2}$ satisfying $u=\operatorname{id}_{X} \times u_{V}$. The map $u$ induces the maps

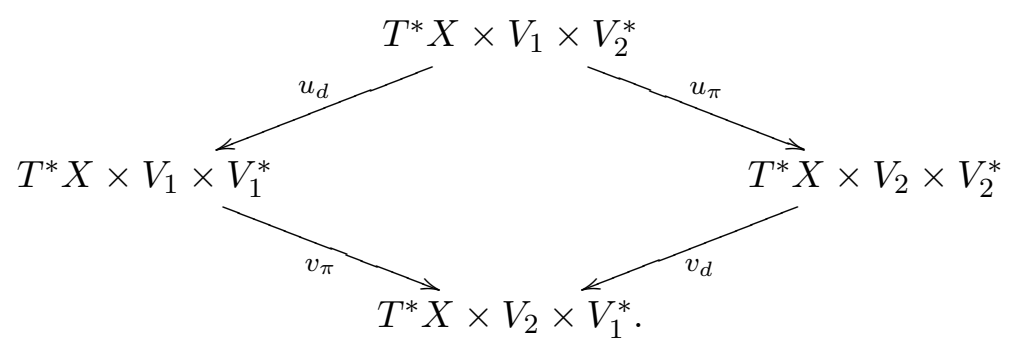

Note that for a subset $A$ of $T^{*}\left(X \times V_{1}\right)$, we have $u_{\pi}\left(u_{d}^{-1}(A)\right)=v_{d}^{-1}\left(v_{\pi}(A)\right)$.

Definition 2.8. Let $u: X \times V_{1} \rightarrow X \times V_{2}$ be a constant linear map and $A \subset T^{*}\left(X \times V_{1}\right)$ be a closed subset. One sets

$$
u_{\sharp}(A):=v_{d}^{-1}\left(\overline{v_{\pi}(A)}\right) .
$$

Proposition 2.9 ([Tam18, Lem. 3.3] and [GS14, Thm. 1.16]). Let $u: X \times V_{1} \rightarrow X \times V_{2}$ be a constant linear map and $F \in \mathbf{D}^{\mathrm{b}}\left(X \times V_{1}\right)$. Then

$$
\mathrm{SS}\left(R u_{*} F\right) \cup \mathrm{SS}\left(R u_{!} F\right) \subset u_{\sharp}(\mathrm{SS}(F)) .
$$




\subsection{Morse-Bott inequality for sheaves ([ST92])}

In this subsection, we give the Morse-Bott inequality for sheaves, which is a slight generalization of the Morse inequality for sheaves by Kashiwara-Schapira [KS90, Prop. 5.4.20] and was proved by Schapira-Tose ST92]. For a bounded complex $W$ of k-vector spaces with finite-dimensional cohomology and $k \in \mathbb{Z}$, we set

$$
b_{k}(W):=\operatorname{dim} H^{k}(W) .
$$

Let $F \in \mathbf{D}^{\mathrm{b}}(X)$ and $\varphi: X \rightarrow \mathbb{R}$ be a $C^{\infty}$-function. We set

$$
\Gamma_{d \varphi}:=\{(x ; d \varphi(x)) \mid x \in X\} \subset T^{*} X .
$$

We consider the following assumptions:

(1) $\operatorname{Supp}(F) \cap \varphi^{-1}((-\infty, t])$ is compact for any $t \in \mathbb{R}$,

(2) the set $\varphi\left(\pi\left(\operatorname{SS}(F) \cap \Gamma_{d \varphi}\right)\right)$ is finite, say $\left\{c_{1}, \ldots, c_{N}\right\}$ with $c_{1}<\cdots<c_{N}$,

(3) the object

$$
W_{i}:=R \Gamma\left(\varphi^{-1}\left(c_{i}\right) ;\left.R \Gamma_{\left\{\varphi \geq c_{i}\right\}}(F)\right|_{\varphi^{-1}\left(c_{i}\right)}\right)
$$

has finite-dimensional cohomology for any $i=1, \ldots, N$.

Theorem 2.10 ([ST92, Thm. 1.1], see also [KS90, Prop. 5.4.20]). Assume that (1)-(3) are satisfied. Then

(i) $R \Gamma(X ; F)$ has finite-dimensional cohomology,

(ii) one has

$$
b_{k}(R \Gamma(X ; F)) \leq \sum_{i=1}^{N} b_{k}\left(W_{i}\right)
$$

for any $k \in \mathbb{Z}$.

Note that [ST92, Thm. 1.1] is a stronger result than Theorem 2.10, In this paper, we only use the weaker inequality (2.14). The proof is the same as [KS90, Prop. 5.4.20], since

$$
R \Gamma_{[t,+\infty)}\left(R \varphi_{*} F\right)_{t} \simeq R \Gamma\left(\varphi^{-1}(t) ;\left.R \Gamma_{\{\varphi \geq t\}}(F)\right|_{\varphi^{-1}(t)}\right) .
$$

\subsection{Kernels ([KS90, §3.6])}

For $i=1,2,3$, let $X_{i}$ be a manifold. We write $X_{i j}:=X_{i} \times X_{j}$ and $X_{123}:=X_{1} \times X_{2} \times X_{3}$ for short. We use the same symbol $q_{i}$ for the projections $X_{i j} \rightarrow X_{i}$ and $X_{123} \rightarrow X_{i}$. We also denote by $q_{i j}$ the projection $X_{123} \rightarrow X_{i j}$. Similarly, we denote by $p_{i j}$ the projection $T^{*} X_{123} \rightarrow T^{*} X_{i j}$. One denotes by $p_{12^{a}}$ the composite of $p_{12}$ and the antipodal map on $T^{*} X_{2}$.

Let $A \subset T^{*} X_{12}$ and $B \subset T^{*} X_{23}$. We set

$$
A \circ B:=p_{13}\left(p_{12^{a}}^{-1} A \cap p_{23}^{-1} B\right) \subset T^{*} X_{13} .
$$


We define the composition of kernels as follows:

$$
\begin{aligned}
\stackrel{\circ}{X_{2}}: \mathbf{D}^{\mathrm{b}}\left(X_{12}\right) \times \mathbf{D}^{\mathrm{b}}\left(X_{23}\right) & \rightarrow \mathbf{D}^{\mathrm{b}}\left(X_{13}\right) \\
\left(K_{12}, K_{23}\right) & \mapsto K_{12} \underset{X_{2}}{\circ} K_{23}:=R q_{13 !}\left(q_{12}^{-1} K_{12} \otimes q_{23}^{-1} K_{23}\right) .
\end{aligned}
$$

If there is no risk of confusion, we simply write $\circ$ instead of $\underset{X_{2}}{\circ}$. By Theorem 2.5 and estimates of the microsupports of tensor products (see [KS90, Prop. 5.4.14]), we have the following proposition.

Proposition 2.11. Let $K_{i j} \in \mathbf{D}^{\mathrm{b}}\left(X_{i j}\right)$ and set $\Lambda_{i j}:=\operatorname{SS}\left(K_{i j}\right) \subset T^{*} X_{i j}(i j=12,23)$. Assume

(1) $q_{13}$ is proper on $q_{12}^{-1} \operatorname{Supp}\left(K_{12}\right) \cap q_{23}^{-1} \operatorname{Supp}\left(K_{23}\right)$,

(2) $p_{12^{a}}^{-1} \Lambda_{12} \cap p_{23}^{-1} \Lambda_{23} \cap\left(T_{X_{1}}^{*} X_{1} \times T^{*} X_{2} \times T_{X_{3}}^{*} X_{3}\right) \subset T_{X_{123}}^{*} X_{123}$.

Then

$$
\mathrm{SS}\left(K_{12} \underset{X_{2}}{\circ} K_{23}\right) \subset \Lambda_{12} \circ \Lambda_{23}
$$

\subsection{Microlocalization and $\mu h o m$ functors ([KS90, §4.3, §4.4])}

Let $M$ be a closed submanifold of $X$. The microlocalization functor along $M$ is a functor $\mu_{M}: \mathbf{D}^{\mathrm{b}}(X) \rightarrow \mathbf{D}^{\mathrm{b}}\left(T_{M}^{*} X\right)$ (see [KS90, $\left.\S 4.3\right]$ for more details). Microlocalization is related to local cohomology as follows. Let $p \in T^{*} X$ and $\varphi: X \rightarrow \mathbb{R}$ be a $C^{\infty}$-function such that $\varphi(\pi(p))=0$ and $d \varphi(\pi(p))=p$. Then, for $F \in \mathbf{D}^{\mathrm{b}}(X)$, we have

$$
R \Gamma_{\{\varphi \geq 0\}}(F)_{\pi(p)} \simeq \mu_{\varphi^{-1}(0)}(F)_{p} .
$$

Under suitable assumptions, the functoriality of microlocalization with respect to proper direct images and non-characteristic inverse images holds as follows:

Proposition 2.12 ([KS90, Prop. 4.3.4 and Cor. 6.7.3]). Let $f: X \rightarrow Y$ be a morphism of manifolds. Moreover, let $N$ be a closed submanifold of $Y$ and assume that $M=f^{-1}(N)$ is also a closed submanifold of $X$. Denote by $f_{M d}: M \times_{N} T_{N}^{*} Y \rightarrow T_{M}^{*} X$ the morphism induced by $f_{d}$ and by $f_{M \pi}: M \times_{N} T_{N}^{*} Y \rightarrow T_{N}^{*} Y$ the morphism induced by $f_{\pi}$ (see (2.1)).

(i) Let $F \in \mathbf{D}^{\mathrm{b}}(X)$. Assume that $f$ is proper on $\operatorname{Supp}(F)$ and $f_{M d}: M \times_{N} T_{N}^{*} Y \rightarrow T_{M}^{*} X$ is surjective. Then

$$
R f_{M \pi !} f_{M d}^{-1} \mu_{M}(F) \stackrel{\sim}{\rightarrow} \mu_{N}\left(R f_{*} F\right) .
$$

(ii) Let $G \in \mathbf{D}^{\mathrm{b}}(Y)$. Assume that $f$ is non-characteristic for $\operatorname{SS}(F)$ and $\left.f\right|_{M}: M \rightarrow N$ is a submersion. Then

$$
\mu_{M}\left(f^{!} G\right) \stackrel{\sim}{\rightarrow} R f_{M d_{*}} f_{M \pi}^{!} \mu_{N}(G) .
$$

We also recall the functor $\mu$ hom. Let $q_{1}, q_{2}: X \times X \rightarrow X$ be the projections. We identify $T_{\Delta_{X}}^{*}(X \times X)$ with $T^{*} X$ through the first projection $(x, x ; \xi,-\xi) \mapsto(x ; \xi)$.

Definition 2.13 ([KS90, Def. 4.4.1]). For $F, G \in \mathbf{D}^{\mathrm{b}}(X)$, one defines

$$
\mu h o m(F, G):=\mu_{\Delta_{X}} R \mathcal{H o m}\left(q_{2}^{-1} F, q_{1}^{!} G\right) \in \mathbf{D}^{\mathrm{b}}\left(T^{*} X\right) .
$$


Proposition 2.14 ([ KS90, Prop. 4.4.2 and Prop. 4.4.3]). Let $F, G \in \mathbf{D}^{\mathrm{b}}(X)$.

(i) $R \pi_{*} \operatorname{\mu hom}(F, G) \simeq R \mathcal{H o m}(F, G)$.

(ii) If $F$ is cohomologically constructible (see [KS90, §3.4] for the definition), then $R \pi ! \mu h o m(F, G) \simeq R \mathcal{H o m}\left(F, \mathbf{k}_{X}\right) \otimes G$.

(iii) For a closed submanifold $M$ of $X, \mu h o m\left(\mathbf{k}_{M}, F\right) \simeq i_{*} \mu_{M}(F)$, where $i: T_{M}^{*} X \rightarrow T^{*} X$ is the embedding.

Proposition 2.15 ([ KS90, Cor. 5.4.10 and Cor. 6.4.3]). Let $F, G \in \mathbf{D}^{\mathrm{b}}(X)$. Then

$$
\begin{aligned}
\operatorname{Supp}(\mu h o m(F, G)) & \subset \mathrm{SS}(F) \cap \operatorname{SS}(G), \\
\operatorname{SS}(\mu h o m(F, G)) & \subset-\mathbf{h}^{-1}(C(\operatorname{SS}(G), \operatorname{SS}(F))),
\end{aligned}
$$

where $C\left(S_{1}, S_{2}\right)$ is the normal cone and $\mathbf{h}: T^{*} T^{*} X \stackrel{\sim}{\rightarrow} T T^{*} X$ is the Hamiltonian isomorphism (see Subsection 2.1).

Proposition 2.16. Let $\varphi: X \rightarrow \mathbb{R}$ be a $C^{\infty}$-function and assume that $d \varphi(x) \neq 0$ for any $x \in \varphi^{-1}(0)$. Set $M:=\varphi^{-1}(0)$ and define an open subset $T_{M}^{*+} X$ of $T_{M}^{*} X$ by

$$
T_{M}^{*+} X:=\{(x ; \lambda d \varphi(x)) \mid x \in M, \lambda>0\} .
$$

Moreover, denote by $\pi_{M+}: T_{M}^{*+} X \rightarrow M$ the projection. Let $F \in \mathbf{D}^{\mathrm{b}}(X)$. Then

$$
\left.\left.\left.R \Gamma_{\{\varphi \geq 0\}}(F)\right|_{M} \simeq R \pi_{M+*} \mu h o m\left(\mathbf{k}_{\{\varphi \geq 0\}}, F\right)\right|_{T_{M}^{*+} X} \simeq R \pi_{M+*} \mu_{M}(F)\right|_{T_{M}^{*+} X} .
$$

In particular,

$$
R \Gamma\left(M ;\left.R \Gamma_{\{\varphi \geq 0\}}(F)\right|_{M}\right) \simeq R \Gamma\left(T_{M}^{*+} X ;\left.\mu_{M}(F)\right|_{T_{M}^{*+} X}\right) .
$$

Proof. Consider the distinguished triangle

$$
\left.R \pi_{!} \mu h o m\left(\mathbf{k}_{\{\varphi \geq 0\}}, F\right) \rightarrow R \pi_{*} \mu h o m\left(\mathbf{k}_{\{\varphi \geq 0\}}, F\right) \rightarrow R \stackrel{\circ}{*}_{*} \mu h o m\left(\mathbf{k}_{\{\varphi \geq 0\}}, F\right)\right|_{T^{*} X} \stackrel{+1}{\rightarrow} .
$$

By Proposition 2.15, $\operatorname{Supp}\left(\left.\mu h o m\left(\mathbf{k}_{\{\varphi \geq 0\}}, F\right)\right|_{T^{*} X}\right) \subset T_{M}^{*+} X$. Hence we have

$$
\left.R \pi_{*}^{\circ} \mu h o m\left(\mathbf{k}_{\{\varphi \geq 0\}}, F\right)\right|_{\overbrace{}^{*} X} \simeq\left(\left.R \pi_{M+*} \mu h o m\left(\mathbf{k}_{\{\varphi \geq 0\}}, F\right)\right|_{T_{M}^{*+} X}\right)_{M} .
$$

On the other hand, since $\mathbf{k}_{\{\varphi \geq 0\}}$ is cohomologically constructible, by Proposition 2.14(i) and (ii), we get

$$
\begin{aligned}
& R \pi_{!} \mu h o m\left(\mathbf{k}_{\{\varphi \geq 0\}}, F\right) \simeq R \mathcal{H o m}\left(\mathbf{k}_{\{\varphi \geq 0\}}, \mathbf{k}_{X}\right) \otimes F \simeq R \Gamma_{\{\varphi \geq 0\}}\left(\mathbf{k}_{X}\right) \otimes F \\
& R \pi_{*} \mu h o m\left(\mathbf{k}_{\{\varphi \geq 0\}}, F\right) \simeq R \mathcal{H o m}\left(\mathbf{k}_{\{\varphi \geq 0\}}, F\right) \simeq R \Gamma_{\{\varphi \geq 0\}}(F) .
\end{aligned}
$$

Since $\left.R \Gamma_{\{\varphi \geq 0\}}\left(\mathbf{k}_{X}\right)\right|_{M} \simeq 0$, restricting the distinguished triangle (2.27) to $M$, we obtain the first isomorphism in (2.25). Moreover since $\operatorname{SS}\left(\mathbf{k}_{\{\varphi>0\}}\right) \cap T_{M}^{*+} X=\emptyset$, by Proposition 2.15, we have

$$
\left.\left.\mu h o m\left(\mathbf{k}_{\{\varphi \geq 0\}}, F\right)\right|_{T_{M}^{*+} X} \stackrel{\sim}{\rightarrow} \mu h o m\left(\mathbf{k}_{\{\varphi=0\}}, F\right)\right|_{T_{M}^{*+} X} .
$$

Thus the second isomorphism in (2.25) follows from Proposition 2.14(iii). 


\subsection{Simple sheaves and quantized contact transformations ([KS90, §7.5])}

Let $\Lambda \subset \stackrel{\circ}{T}^{*} X$ be a locally closed conic Lagrangian submanifold and $p \in \Lambda$. Simple sheaves along $\Lambda$ at $p$ are defined in [KS90, Def. 7.5.4]. In this subsection we recall them.

Let $\varphi: X \rightarrow \mathbb{R}$ be a $C^{\infty}$-function such that $\varphi(\pi(p))=0$ and $\Gamma_{d \varphi}$ intersects $\Lambda$ transversally at $p$. For $p \in \Gamma_{d \varphi} \cap \Lambda$, we define the following Lagrangian subspaces in $T_{p} T^{*} X$ :

$$
\lambda_{\infty}(p):=T_{p}\left(T_{\pi(p)}^{*} X\right), \quad \lambda_{\Lambda}(p):=T_{p} \Lambda, \quad \lambda_{\varphi}(p):=T_{p} \Gamma_{d \varphi} .
$$

Here, our notation $\lambda_{\infty}(p)$ is different from that of [KS90], where the authors write $\lambda_{0}(p)$ for $T_{p}\left(T_{\pi(p)}^{*} X\right)$. In this paper we do not use the symbol $\lambda_{0}(p)$. We briefly recall the definition of the inertia index of a triple of Lagrangian subspaces (see [KS90, §A.3]). Let $(E, \sigma)$ be a symplectic vector space and $\lambda_{1}, \lambda_{2}, \lambda_{3}$ be three Lagrangian subspaces of $E$. We define a quadratic form $q$ on $\lambda_{1} \oplus \lambda_{2} \oplus \lambda_{3}$ by $q\left(v_{1}, v_{2}, v_{3}\right)=\sigma\left(v_{1}, v_{2}\right)+\sigma\left(v_{2}, v_{3}\right)+\sigma\left(v_{3}, v_{1}\right)$. Then the inertia index $\tau_{E}\left(\lambda_{\infty}, \lambda_{1}, \lambda_{3}\right)$ of the triple is defined as the signature of $q$. Using the inertia index and the notation (2.31), one sets

$$
\tau_{\varphi}=\tau_{p, \varphi}:=\tau_{T_{p} T^{*} X}\left(\lambda_{\infty}(p), \lambda_{\Lambda}(p), \lambda_{\varphi}(p)\right) .
$$

Proposition 2.17 ([KS90, Prop. 7.5.3]). For $i=1,2$, let $\varphi_{i}: X \rightarrow \mathbb{R}$ be a $C^{\infty}$-function such that $\varphi_{i}(\pi(p))=0$ and $\Gamma_{d \varphi_{i}}$ intersects $\Lambda$ transversally at $p$. Let $F \in \mathbf{D}^{\mathrm{b}}(X)$ and assume that $\mathrm{SS}(F) \subset \Lambda$ in a neighborhood of $p$. Then

$$
R \Gamma_{\left\{\varphi_{1} \geq 0\right\}}(F)_{\pi(p)} \simeq R \Gamma_{\left\{\varphi_{2} \geq 0\right\}}(F)_{\pi(p)}\left[\frac{1}{2}\left(\tau_{\varphi_{2}}-\tau_{\varphi_{1}}\right)\right] .
$$

Definition 2.18 ([KS90, Def. 7.5.4]). In the situation of Proposition 2.17, $F$ is said to have microlocal type $L \in \mathbf{D}^{\mathrm{b}}(\operatorname{Mod}(\mathbf{k}))$ with shift $d \in \frac{1}{2} \mathbb{Z}$ at $p$ if

$$
R \Gamma_{\{\varphi \geq 0\}}(F)_{\pi(p)} \simeq L\left[d-\frac{1}{2} \operatorname{dim} X-\frac{1}{2} \tau_{\varphi}\right]
$$

for some (hence for any) $C^{\infty}$-function $\varphi$ such that $\varphi(\pi(p))=0$ and $\Gamma_{d \varphi}$ intersects $\Lambda$ transversally at $p$. Moreover, if $L \simeq \mathbf{k}, F$ is said to be simple along $\Lambda$ at $p$. If $F$ is simple at all points of $\Lambda$, one says that $F$ is simple along $\Lambda$.

One can prove that if $F \in \mathbf{D}^{\mathrm{b}}(X)$ is simple along $\Lambda$, then $\left.\mu h o m(F, F)\right|_{\Lambda} \simeq \mathbf{k}_{\Lambda}$. When $\Lambda$ is a conormal bundle to a closed submanifold $M$ of $X$ in a neighborhood of $p$, that is, $\left.\pi\right|_{\Lambda}: \Lambda \rightarrow X$ has constant rank, then $F \in \mathbf{D}^{\mathrm{b}}(X)$ is simple along $\Lambda$ at $p$ if $F \simeq \mathbf{k}_{M}[d]$ in $\mathbf{D}^{\mathrm{b}}(X ; p)$ for some $d \in \mathbb{Z}$.

Example 2.19. Let $X=\mathbb{R}^{n+1}$ and consider the hyperplane $M=\mathbb{R}^{n} \times\{0\}$. Then $\mathbf{k}_{M}$ is simple with shift $1 / 2$ along $T_{M}^{*} X$.

We also recall the notion of quantized contact transformations. Let $\chi: T^{*} X \supset \Omega_{1} \stackrel{\sim}{\rightarrow}$ $\Omega_{2} \subset T^{*} X$ be a contact transformation. A quantized contact transformation associated with $\chi$ is a kernel $K \in \mathbf{D}^{\mathrm{b}}(X \times X)$ which is simple along $\left(\operatorname{id}_{X} \times a\right)^{-1} \operatorname{Graph}(\chi)$ in $\Omega_{2} \times \Omega_{1}^{a}$ and satisfies some properties (see [KS90, §7.2] for details). A quantized contact transformation $K$ induces an equivalence of categories

$$
K \circ(*): \mathbf{D}^{\mathrm{b}}\left(X ; \Omega_{1}\right) \stackrel{\sim}{\rightarrow} \mathbf{D}^{\mathrm{b}}\left(X ; \Omega_{2}\right) .
$$

Proposition 2.20 ([KS90, Thm. 7.2.1]). Let $K \in \mathbf{D}^{\mathrm{b}}(X \times X)$ be a quantized contact transformation associated with a contact transformation $\chi: T^{*} X \supset \Omega_{1} \stackrel{\sim}{\rightarrow} \Omega_{2} \subset T^{*} X$. Moreover, let $F, G \in \mathbf{D}^{\mathrm{b}}\left(X ; \Omega_{1}\right)$. Then

$$
\left.\operatorname{\mu hom}(K \circ F, K \circ G)\right|_{\Omega_{2}} \simeq \chi_{*}\left(\left.\mu h o m(F, G)\right|_{\Omega_{1}}\right) .
$$


The behavior of the shift of a simple sheaf under a quantized contact transformation is described by the inertia index.

Proposition 2.21 ([KS90, Prop. 7.5.6 and Thm. 7.5.11]). Let $F \in \mathbf{D}^{\mathrm{b}}(X)$ and assume that $F$ is simple with shift $d$ along $\Lambda$ at $p$. Let $\chi: T^{*} X \supset \Omega_{1} \stackrel{\sim}{\rightarrow} \Omega_{2} \subset T^{*} X$ be a contact transformation defined in a neighborhood of $p$ and $K \in \mathbf{D}^{\mathrm{b}}(X \times X)$ be a quantized contact transformation associated with $\chi$. Assume that $K$ is simple with shift $d^{\prime}$ along $\left(\operatorname{id}_{X} \times a\right)^{-1} \operatorname{Graph}(\chi)$ at $\left(\chi(p), p^{a}\right)$. Then $K \circ F$ is simple with shift $d+d^{\prime}-\delta$ along $\chi(\Lambda)$ at $\chi(p)$, where

$$
\delta:=\frac{1}{2} \operatorname{dim} X+\frac{1}{2} \tau\left(\lambda_{\infty}(p), \lambda_{\Lambda}(p), \chi^{-1}\left(\lambda_{\infty}(\chi(p))\right)\right) .
$$

\section{Sheaf quantization and Tamarkin's non-displaceability theorem}

In what follows, until the end of the paper, let $M$ be a non-empty compact connected manifold without boundary.

In this section, we review Tamarkin's approach to non-displaceability problems in symplectic geometry based on microlocal sheaf theory. We also review sheaf quantization of Hamiltonian isotopies and compact exact Lagrangian submanifolds in cotangent bundles.

\subsection{Sheaf quantization of Hamiltonian isotopies ([GKS12])}

Guillermou-Kashiwara-Schapira [GKS12] constructed sheaf quantizations of Hamiltonian isotopies. Since the microsupports of sheaves are conic subsets of cotangent bundles, microlocal sheaf theory is related to the exact (homogeneous) symplectic structures rather than the symplectic structures of cotangent bundles. In order to treat non-homogeneous Hamiltonian isotopies and non-conic Lagrangian submanifolds, an important trick is to add a variable and "conify" them, which is an idea of Tamarkin's.

Denote by $(x ; \xi)$ a local homogeneous symplectic coordinate system on $T^{*} M$ and by $(t ; \tau)$ the homogeneous symplectic coordinate system on $T^{*} \mathbb{R}$. We set $\Omega_{+}:=\{\tau>0\} \subset$ $T^{*}(M \times \mathbb{R})$ and define the map

$$
\begin{aligned}
& \rho: \Omega_{+} \longrightarrow T^{*} M \\
& (x, t ; \xi, \tau) \longmapsto(x ; \xi / \tau) \text {. }
\end{aligned}
$$

Let $I$ be an open interval in $\mathbb{R}$ containing 0 and $\phi=\left(\phi_{s}\right)_{s \in I}: T^{*} M \times I \rightarrow T^{*} M$ be a Hamiltonian isotopy with compact support. Note that $\phi$ is the identity for $s=0$ : $\phi_{0}=\operatorname{id}_{T^{*} M}$. Then one can construct a homogeneous Hamiltonian isotopy $\widehat{\phi}: \stackrel{\circ}{T}^{*}(M \times$ $\mathbb{R}) \times I \rightarrow T^{*}(M \times \mathbb{R})$ such that the following diagram commutes:

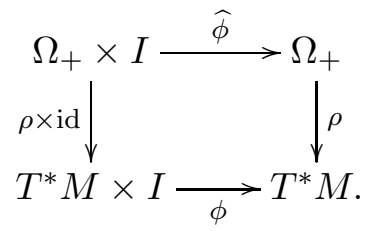

Here $\widehat{\phi}$ is called a homogeneous Hamiltonian isotopy if it is a Hamiltonian isotopy whose Hamiltonian function $\widehat{H}$ is homogeneous of degree $1: \widehat{H}_{s}(x, t ; c \xi, c \tau)=c \cdot \widehat{H}_{s}(x, t ; \xi, \tau)$ for 
any $c>0$. See [GKS12, Sect. A.3] for more details. For simplicity, we set $N:=M \times \mathbb{R}$ and consider a homogeneous Hamiltonian isotopy $\widehat{\phi}=\left(\widehat{\phi}_{s}\right)_{s}: \stackrel{\circ}{ }^{*} N \times I \rightarrow T^{*} N$ and the associated homogeneous Hamiltonian $\widehat{H}: \stackrel{\circ}{T}^{*} N \times I \rightarrow \mathbb{R}$. We define a conic Lagrangian submanifold $\Lambda_{\widehat{\phi}} \subset \stackrel{\circ}{T}^{*} N \times \stackrel{\circ}{T}^{*} N \times T^{*} I$ by

$$
\Lambda_{\widehat{\phi}}:=\left\{\left(\widehat{\phi}_{s}(y ; \eta),(y ;-\eta),\left(s ;-\widehat{H}_{s} \circ \widehat{\phi}_{s}(y ; \eta)\right)\right) \mid(y ; \eta) \in \stackrel{\circ}{T}^{*} N, s \in I\right\} .
$$

Note that

$$
\Lambda_{\widehat{\phi}} \circ T_{s}^{*} I=\left\{\left(\widehat{\phi}_{s}(y ; \eta),(y ;-\eta)\right) \mid(y ; \eta) \in \stackrel{\circ}{T}^{*} N\right\} \subset \stackrel{\circ}{T}^{*} N \times \stackrel{\circ}{T}^{*} N
$$

for any $s \in I$ (see (2.16) for the definition of $A \circ B$ ).

Theorem 3.1 ([GKS12, Thm. 4.3]). For a homogeneous Hamiltonian isotopy $\widehat{\phi}: \stackrel{T}{T}^{*} N \times$ $I \rightarrow \stackrel{\circ}{T}^{*} N$, there exists a unique object $K \in \mathbf{D}^{\mathrm{lb}}(N \times N \times I)$ satisfying the following conditions:

(1) $\operatorname{SS}(K) \subset \Lambda_{\widehat{\phi}} \cup T_{N \times N \times I}^{*}(N \times N \times I)$,

(2) $\left.K\right|_{N \times N \times\{0\}} \simeq \mathbf{k}_{\Delta_{N}}$, where $\Delta_{N}$ is the diagonal of $N \times N$.

Moreover $K$ is simple along $\Lambda_{\widehat{\phi}}$ and both projections $\operatorname{Supp}(K) \rightarrow N \times I$ are proper.

The object $K$ is called the sheaf quantization of $\widehat{\phi}$. For any $s \in I, \operatorname{Sis}\left(\left.K\right|_{N \times N \times\{s\}}\right) \subset$ $\Lambda_{\widehat{\phi}} \circ T_{s}^{*} I$ and $\left.K\right|_{N \times N \times\{s\}}$ is a quantized contact transformation associated with $\widehat{\phi}_{s}: \Omega_{+} \stackrel{\sim}{\rightarrow}$ $\Omega_{+}$.

\subsection{Tamarkin's non-displaceability theorem ([Tam18, GS14])}

A diffeomorphism $\psi: T^{*} M \rightarrow T^{*} M$ is said to be a Hamiltonian diffeomorphism if there exists a Hamiltonian isotopy with compact support $\phi=\left(\phi_{s}\right)_{s}: T^{*} M \times[0,1] \rightarrow T^{*} M$ such that $\phi_{1}=\psi$ and $\phi_{0}=\operatorname{id}_{T^{*} M}$. Two compact subsets $A$ and $B$ of $T^{*} M$ are said to be mutually non-displaceable if $A \cap \psi(B) \neq \emptyset$ for any Hamiltonian diffeomorphism $\psi: T^{*} M \rightarrow T^{*} M$. The non-displaceability problem is to determine whether or not given two compact subsets are mutually non-displaceable. Tamarkin [Tam18] (see also Guillermou-Schapira GS14]) considered some categories consisting of sheaves on $M \times \mathbb{R}$ and deduced a criterion for non-displaceability using them.

We set $\Omega_{+}:=\{\tau>0\} \subset T^{*}(M \times \mathbb{R})$ as before, where $(t ; \tau)$ denotes the homogeneous symplectic coordinate system on $T^{*} \mathbb{R}$. We define the maps

$$
\begin{gathered}
\tilde{q}_{1}, \tilde{q}_{2}, s_{\mathbb{R}}: M \times \mathbb{R} \times \mathbb{R} \longrightarrow M \times \mathbb{R}, \\
\tilde{q}_{1}\left(x, t_{1}, t_{2}\right)=\left(x, t_{1}\right), \tilde{q}_{2}\left(x, t_{1}, t_{2}\right)=\left(x, t_{2}\right), s_{\mathbb{R}}\left(x, t_{1}, t_{2}\right)=\left(x, t_{1}+t_{2}\right) .
\end{gathered}
$$

If there is no risk of confusion, we simply write $s$ for $s_{\mathbb{R}}$. We also define the involution

$$
i: M \times \mathbb{R} \rightarrow M \times \mathbb{R},(x, t) \longmapsto(x,-t) .
$$

Definition 3.2. For $F, G \in \mathbf{D}^{\mathrm{b}}(M \times \mathbb{R})$, one sets

$$
\begin{aligned}
F \star G & :=R s_{!}\left(\tilde{q}_{1}^{-1} F \otimes \tilde{q}_{2}^{-1} G\right), \\
\mathcal{H o m}{ }^{\star}(F, G) & :=R \tilde{q}_{1 *} R \mathcal{H o m}\left(\tilde{q}_{2}^{-1} F, s^{!} G\right) \\
& \simeq R s_{*} R \mathcal{H o m}\left(\tilde{q}_{2}^{-1} i^{-1} F, \tilde{q}_{1}^{!} G\right) .
\end{aligned}
$$


Note that the functor $\star$ is a left adjoint to $\mathcal{H o m}^{\star}$. The functor

$$
\mathbf{k}_{M \times[0,+\infty)} \star(*): \mathbf{D}^{\mathrm{b}}(M \times \mathbb{R}) \longrightarrow \mathbf{D}^{\mathrm{b}}(M \times \mathbb{R})
$$

defines a projector on the left orthogonal ${ }^{\perp} \mathbf{D}_{\{\tau \leq 0\}}^{\mathrm{b}}(M \times \mathbb{R})$. By using this projector, Tamarkin proved that the localized category $\mathbf{D}^{\mathrm{b}}\left(M \times \mathbb{R} ; \Omega_{+}\right)$is equivalent to the left orthogonal ${ }^{\perp} \mathbf{D}_{\{\tau \leq 0\}}^{\mathrm{b}}(M \times \mathbb{R})$ :

$$
\mathbf{D}^{\mathrm{b}}\left(M \times \mathbb{R} ; \Omega_{+}\right)=\mathbf{D}^{\mathrm{b}}(M \times \mathbb{R}) / \mathbf{D}_{\{\tau \leq 0\}}^{\mathrm{b}}(M \times \mathbb{R}) \stackrel{\sim}{\rightarrow}{ }^{\perp} \mathbf{D}_{\{\tau \leq 0\}}^{\mathrm{b}}(M \times \mathbb{R}) .
$$

Definition 3.3 ([Tam18]). One defines

$$
\mathcal{D}(M):=\mathbf{D}^{\mathrm{b}}\left(M \times \mathbb{R} ; \Omega_{+}\right) \simeq{ }^{\perp} \mathbf{D}_{\{\tau \leq 0\}}^{\mathrm{b}}(M \times \mathbb{R}) .
$$

For a compact subset $A$ of $T^{*} M$, one also defines a full subcategory $\mathcal{D}_{A}(M)$ of $\mathcal{D}(M)$ by

$$
\mathcal{D}_{A}(M):=\mathbf{D}_{\rho^{-1}(A)}^{\mathrm{b}}\left(M \times \mathbb{R} ; \Omega_{+}\right) .
$$

For an object in $\mathcal{D}(M)$, we take the canonical representative in ${ }^{\perp} \mathbf{D}_{\{\tau \leq 0\}}^{\mathrm{b}}(M \times \mathbb{R})$ via the projector unless otherwise specified.

Proposition 3.4 ([GS14, Lem. 3.18]). Let $F, G \in \mathcal{D}(M)$. Then

$$
\operatorname{Hom}_{\mathcal{D}(M)}(F, G) \simeq H^{0} R \Gamma_{M \times[0,+\infty)}\left(M \times \mathbb{R} ; \mathcal{H o m}^{\star}(F, G)\right) .
$$

The following separation theorem is due to Tamarkin Tam18].

Theorem 3.5 ([Tam18, Thm. 3.2] and [GS14, Thm. 3.28]). Let $A$ and $B$ be compact subsets of $T^{*} M$ and assume that $A \cap B=\emptyset$. Then for $F \in \mathcal{D}_{A}(M)$ and $G \in \mathcal{D}_{B}(M)$, one has $\operatorname{Hom}_{\mathcal{D}(M)}(F, G) \simeq 0$.

Proof. We give the outline of the proof. Denote by $t: M \times \mathbb{R} \rightarrow \mathbb{R}$ the function $(x, t) \mapsto t$. Recall the notation $\Gamma_{d t}=\{(x, t ; 0,1)\} \subset T^{*}(M \times \mathbb{R})$. Then one can show that

$$
\Gamma_{d t} \cap \operatorname{SS}\left(R \Gamma_{M \times[0,+\infty)} \mathcal{H o m}{ }^{\star}(F, G)\right)=\emptyset .
$$

Hence by Proposition 3.4 and the microlocal Morse lemma (Proposition 2.3), we have the conclusion.

Using sheaf quantization of Hamiltonian isotopies, we can define Hamiltonian deformations in the category $\mathcal{D}(M)$ as follows. Let $\psi: T^{*} M \rightarrow T^{*} M$ be a Hamiltonian diffeomorphism and $\phi=\left(\phi_{s}\right)_{s}: T^{*} M \times I \rightarrow T^{*} M$ be a Hamiltonian isotopy with compact support satisfying $\psi=\phi_{1}$, where $I$ is an open interval containing the closed interval $[0,1]$. Let $\widehat{\phi}: \stackrel{\circ}{T}^{*}(M \times \mathbb{R}) \times I \rightarrow \overleftarrow{T}^{*}(M \times \mathbb{R})$ be the associated homogeneous Hamiltonian isotopy and $K \in \mathbf{D}^{\mathrm{lb}}(M \times \mathbb{R} \times M \times \mathbb{R} \times I)$ be the sheaf quantization of $\widehat{\phi}$. Then the composition with $K_{1}:=\left.K\right|_{M \times \mathbb{R} \times M \times \mathbb{R} \times\{1\}} \in \mathbf{D}^{\mathrm{b}}(M \times \mathbb{R} \times M \times \mathbb{R})$ defines a functor

$$
\Psi=K_{1} \circ(*): \mathbf{D}^{\mathrm{b}}(M \times \mathbb{R}) \longrightarrow \mathbf{D}^{\mathrm{b}}(M \times \mathbb{R}),
$$

which induces a functor $\Psi: \mathcal{D}(M) \rightarrow \mathcal{D}(M)$ (see [GS14, Prop. 3.29]) 2). Let $A$ be a compact subset of $T^{*} M$. Then, for any $F \in \mathcal{D}_{A}(M)$, Proposition 2.11 and the commutative diagram (3.2) imply

$$
\operatorname{SS}\left(K_{1} \circ F\right) \cap \Omega_{+} \subset\left(\Lambda_{\widehat{\phi}} \circ T_{1}^{*} I\right) \circ \rho^{-1}(A)=\widehat{\phi}_{1}\left(\rho^{-1}(A)\right) \subset \rho^{-1}(\psi(A)) .
$$

\footnotetext{
${ }^{2}$ Although $\widehat{\phi}$ does not satisfy [GKS12, (3.3)] in general, $\left.K\right|_{M \times \mathbb{R} \times M \times \mathbb{R} \times J}$ is bounded for any relatively compact subinterval $J$ of $I$. The author learned the detailed proof from S. Guillermou. One can prove it using the properness of $\operatorname{Supp}(K) \rightarrow M \times \mathbb{R} \times I$ and the fact that $K \simeq \sigma^{-1} K^{\prime}$, where $K^{\prime} \in \mathbf{D}^{\mathrm{lb}}(M \times M \times \mathbb{R} \times I)$ and $\sigma: M \times \mathbb{R} \times M \times \mathbb{R} \times I \rightarrow M \times M \times \mathbb{R} \times I,\left(x, t, x^{\prime}, t^{\prime}, s\right) \mapsto\left(x, x^{\prime}, t-t^{\prime}, s\right)$.
} 
Hence the functor also induces $\Psi: \mathcal{D}_{A}(M) \rightarrow \mathcal{D}_{\psi(A)}(M)$.

Tamarkin [Tam18] proved the non-displaceability theorem by using the category $\mathcal{D}(M)$ and torsion objects, which we will explain below. Moreover, Guillermou-Schapira [GS14] proved that torsion objects form a triangulated subcategory and introduced the quotient category $\mathcal{T}(M)$, which is invariant under Hamiltonian deformations. For $c \in \mathbb{R}$, we define the translation map

$$
T_{c}: M \times \mathbb{R} \rightarrow M \times \mathbb{R},(x, t) \mapsto(x, t+c) .
$$

For $F \in{ }^{\perp} \mathbf{D}_{\{\tau \leq 0\}}^{\mathrm{b}}(M \times \mathbb{R})$ and $c \in \mathbb{R}_{\geq 0}$, there exists a canonical morphism $\tau_{0, c}(F): F \rightarrow$ $T_{c *} F$.

Definition 3.6 ([Tam18]). An object $F \in{ }^{\perp} \mathbf{D}_{\{\tau \leq 0\}}^{\mathrm{b}}(M \times \mathbb{R})$ is said to be a torsion object if $\tau_{0, c}(F)=0$ for some $c \geq 0$. Denote by $\mathcal{N}_{\text {tor }}$ the subcategory of torsion objects in ${ }^{\perp} \mathbf{D}_{\{\tau \leq 0\}}^{\mathrm{b}}(M \times \mathbb{R}) \simeq \mathcal{D}(M)$.

Let $F \in{ }^{\perp} \mathbf{D}_{\{\tau \leq 0\}}^{\mathrm{b}}(M \times \mathbb{R})$ and assume that $\operatorname{Supp}(F) \subset M \times[a, b]$ for some compact interval $[a, b]$ of $\mathbb{R}$. Then $F$ is a torsion object.

Proposition 3.7 ([GS14, Thm. 5.4]). The subcategory $\mathcal{N}_{\text {tor }}$ is a full triangulated subcategory of $\mathcal{D}(M)$.

Definition 3.8 (GS14, Def. 5.6]). The triangulated category $\mathcal{T}(M)$ is defined as the quotient category of $\mathcal{D}(M)$ by $\mathcal{N}_{\text {tor }}: \mathcal{T}(M):=\mathcal{D}(M) / \mathcal{N}_{\text {tor }}$.

Hom spaces in $\mathcal{T}(M)$ are described as inductive limits of those in $\mathcal{D}(M)$.

Proposition 3.9 ([GS14, Prop. 5.7]). Let $F, G \in \mathcal{D}(M)$. Then

$$
\underset{c \rightarrow+\infty}{\lim _{\longrightarrow}} \operatorname{Hom}_{\mathcal{D}(M)}\left(F, T_{c *} G\right) \stackrel{\sim}{\rightarrow} \operatorname{Hom}_{\mathcal{T}(M)}(F, G) .
$$

The following is the Hamiltonian invariance theorem due to Tamarkin [Tam18].

Theorem 3.10 ([Tam18, Thm. 3.9] and GS14, Thm. 6.1]). Let $\psi: T^{*} M \rightarrow T^{*} M$ be a Hamiltonian diffeomorphism and $\Psi: \mathcal{D}(M) \rightarrow \mathcal{D}(M)$ be the functor associated with $\psi$. Then, for any $F \in \mathcal{D}(M)$, one has

$$
F \simeq \Psi(F) \quad \text { in } \mathcal{T}(M)
$$

Combining Theorem 3.10 with Theorem 3.5 and Proposition 3.9, we can deduce the following non-displaceability theorem.

Theorem 3.11 ([Tam18, Thm. 3.1] and [GS14, Cor. 6.3]). Let $A$ and $B$ be compact subsets of $T^{*} M$. Assume that there exist $F \in \mathcal{D}_{A}(M)$ and $G \in \mathcal{D}_{B}(M)$ such that $\operatorname{Hom}_{\mathcal{T}(M)}(F, G) \neq 0$. Then $A$ and $B$ are mutually non-displaceable.

\subsection{Guillermou's sheaf quantization of compact exact Lagrangian sub- manifolds ([Gui12, Gui16])}

Recall that a Lagrangian submanifold $L$ of $T^{*} M$ is said to be exact if the restriction of the Liouville 1-form $\left.\alpha\right|_{L}$ is exact. Guillermou [Gui12, Gui16] proved the existence of sheaf quantizations of compact exact Lagrangian submanifolds of $T^{*} M$. 
Let $L$ be a compact connected exact Lagrangian submanifold of $T^{*} M$ and choose a primitive of the Liouville 1-form $f: L \rightarrow \mathbb{R}$ satisfying $d f=\left.\alpha\right|_{L}$. We define the conification $\widehat{L}_{f} \subset \Omega_{+}$of $L$ with respect to $f$ by

$$
\widehat{L}_{f}:=\{(x, t ; \tau \xi, \tau) \mid \tau>0,(x ; \xi) \in L, t=-f(x ; \xi)\} .
$$

If there is no risk of confusion, we simply write $\widehat{L}$ instead of $\widehat{L}_{f}$.

Consider the category $\mathbf{D}_{\widehat{L} \cup T_{M \times \mathbb{R}}^{*}(M \times \mathbb{R})}^{\mathrm{b}}(M \times \mathbb{R})$ consisting of sheaves whose microsupports are contained in $\widehat{L} \cup T_{M \times \mathbb{R}}^{*}(M \times \mathbb{R})$. By the compactness of $L$, there is $A \in \mathbb{R}_{>0}$ such that $\widehat{L} \subset T^{*}(M \times(-A, A))$. Hence for any $F \in \mathbf{D}_{\widehat{L} \cup T_{M \times \mathbb{R}}^{*}}^{\mathrm{b}}(M \times \mathbb{R})(M \times \mathbb{R})$, the restrictions $\left.F\right|_{M \times(-\infty,-A)}$ and $\left.F\right|_{M \times(A,+\infty)}$ are locally constant.

Definition 3.12 (Gui12, Def. 20.1] and Gui16, Def. 13.1]). Let $A \in \mathbb{R}_{>0}$ satisfying $\widehat{L} \subset T^{*}(M \times(-A, A))$. For $F \in \mathbf{D}_{\widehat{L} \cup T_{M \times \mathbb{R}}^{*}(M \times \mathbb{R})}^{\mathrm{b}}(M \times \mathbb{R})$, one defines $F_{-}, F_{+} \in \mathbf{D}^{\mathrm{b}}(M)$ by

$$
F_{-}:=\left.F\right|_{M \times\{-t\}}, \quad F_{+}:=\left.F\right|_{M \times\{t\}}
$$

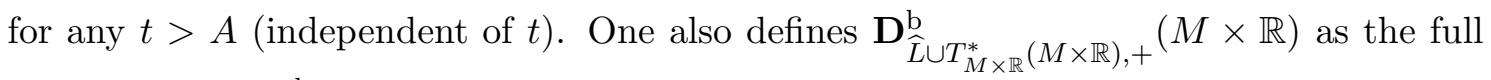

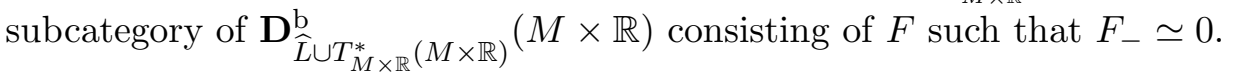

Guillermou [Gui12, Gui16] proved the following existence and uniqueness of sheaf quantizations of compact exact Lagrangian submanifolds.

Theorem 3.13 ([Gui12, Thm. 26.1] and [Gui16, Thm. 18.1]). Let L, $f$, and $\widehat{L}=\widehat{L}_{f}$ be as above.

(i) For any rank 1 locally constant sheaf $\mathcal{L} \in \operatorname{Mod}\left(\mathbf{k}_{M}\right)$, there exists an object $F \in$

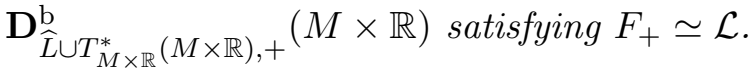

(ii) Moreover $F$ in (i) is unique up to a unique isomorphism and simple along $\widehat{L}$.

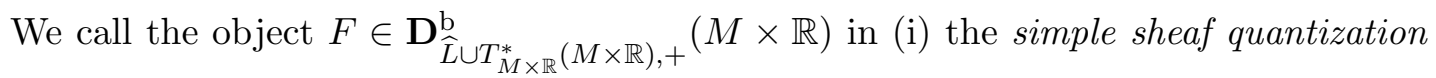
of $\widehat{L}$ with respect to the rank 1 locally constant sheaf $\mathcal{L}$. Moreover if $\mathcal{L}$ is the constant sheaf $\mathbf{k}_{M}$, that is, $F_{+} \simeq \mathbf{k}_{M}$, then $F$ is said to be the canonical sheaf quantization of $\widehat{L}$. Note that the simple sheaf quantization of $\widehat{L}$ with respect to $\mathcal{L}$ is of the form $F \otimes q_{M}^{-1} \mathcal{L}$, where $F$ is the canonical sheaf quantization and $q_{M}: M \times \mathbb{R} \rightarrow M$ is the projection. We sometimes write a sheaf quantization associated with $L$ (and $f$ ) instead of $\widehat{L}$ for simplicity.

\section{Intersections of compact exact Lagrangian submanifolds in cotangent bundles and sheaf quantization}

In this section we study intersections of compact exact Lagrangian submanifolds in cotangent bundles, using Tamarkin's category and Guillermou's sheaf quantizations. In particular, we prove Theorem 1.1, a Morse-Bott-type inequality for clean Lagrangian intersections. Throughout this section, for $i=1,2$ let $L_{i}$ be a compact connected exact Lagrangian submanifold and $f_{i}: L_{i} \rightarrow \mathbb{R}$ be a primitive of the Liouville 1-form satisfying $d f_{i}=\left.\alpha\right|_{L_{i}}$. We denote by $\Lambda_{i}:=\widehat{L}_{i}$ the conification of $L_{i}$ with respect to $f_{i}$. Moreover, let $F_{i} \in \mathbf{D}_{\Lambda_{i} \cup T_{M \times \mathbb{R}}^{*}}^{\mathrm{b}}(M \times \mathbb{R})(M \times \mathbb{R})$ be a simple sheaf quantization of $\Lambda_{i}$. Until the end of Subsection 4.3, we do not assume that $L_{1}$ and $L_{2}$ intersect cleanly. 


\subsection{Non-displaceability of compact exact Lagrangian submanifolds}

In this subsection we prove that the Hom space in $\mathcal{T}(M)$ between the canonical sheaf quantizations associated with compact exact Lagrangian submanifolds is isomorphic to the cohomology of the base manifold $M$. Combined with Theorem 3.11, this implies the non-displaceability.

First we give a preliminary result useful to calculate Hom spaces in $\mathcal{D}(M)$.

Lemma 4.1. Let $L$ be a compact connected exact Lagrangian submanifold of $T^{*} M$ and $\Lambda=\widehat{L}$ be the conification of $L$ with respect to some primitive. Then

$$
\mathbf{D}_{\Lambda \cup T_{M \times \mathbb{R}}^{*}(M \times \mathbb{R}),+}^{\mathrm{b}}(M \times \mathbb{R}) \subset{ }^{\perp} \mathbf{D}_{\{\tau \leq 0\}}^{\mathrm{b}}(M \times \mathbb{R}) .
$$

Proof. By compactness, there exists a constant $B \in \mathbb{R}$ such that $\Lambda \subset T^{*}(M \times(B,+\infty))$. Let $F \in \mathbf{D}_{\Lambda \cup T_{M \times \mathbb{R}}^{*}}^{\mathrm{b}}(M \times \mathbb{R}),+(M \times \mathbb{R})$ and $G \in \mathbf{D}_{\{\tau \leq 0\}}^{\mathrm{b}}(M \times \mathbb{R})$. Since $\Lambda \subset\{\tau>0\}$, by Proposition 2.7, we have $\operatorname{SS}(R \mathcal{H}$ om $(F, G)) \subset\{\tau \leq 0\}$. Applying the microlocal Morse lemma (Proposition 2.3) to $R \mathcal{H o m}(F, G)$ and the function $t: M \times \mathbb{R} \rightarrow \mathbb{R},(x, t) \mapsto t$, we get $R \operatorname{Hom}(F, G) \simeq 0$ by the inclusion $\operatorname{Supp}(R \mathcal{H o m}(F, G)) \subset M \times[B,+\infty)$.

Proposition 4.2. Let $\mathcal{L}_{i}:=\left(F_{i}\right)_{+} \in \operatorname{Mod}\left(\mathbf{k}_{M}\right)$ be the locally constant sheaf of rank 1 associated with the simple sheaf quantization $F_{i}$ for $i=1,2$. Then there exists $c_{0} \in \mathbb{R}_{\geq 0}$ such that $\operatorname{Hom}_{\mathcal{D}(M)}\left(F_{2}, T_{c *} F_{1}[k]\right)$ is isomorphic to $H^{k}\left(M ; \mathcal{L}_{1} \otimes \mathcal{L}_{2}^{\otimes-1}\right)$ for any $c \geq c_{0}$ and $k \in \mathbb{Z}$. In particular,

$$
\operatorname{Hom}_{\mathcal{T}(M)}\left(F_{2}, F_{1}[k]\right) \simeq H^{k}\left(M ; \mathcal{L}_{1} \otimes \mathcal{L}_{2}^{\otimes-1}\right) \quad \text { for any } k \in \mathbb{Z} .
$$

Proof. The proof is very similar to those of [Gui12, Thm. 20.3] and Gui16, Thm. 13.3]. By Lemma 4.1, for any $k \in \mathbb{Z}$, we have

$$
\operatorname{Hom}_{\mathcal{D}(M)}\left(F_{2}, T_{c *} F_{1}[k]\right)=\operatorname{Hom}_{\mathbf{D}^{\mathrm{b}}(M \times \mathbb{R})}\left(F_{2}, T_{c *} F_{1}[k]\right) .
$$

By the compactness of $L_{1}$ and $L_{2}$, there exists $A \in \mathbb{R}_{>0}$ satisfying $\Lambda_{1}, \Lambda_{2} \subset T^{*}(M \times$ $(-A, A))$. Take a sufficiently large $c_{0} \in \mathbb{R}_{\geq 0}$ such that $c_{0}>2 A$. Then, by the isomorphism $\left.F_{2}\right|_{M \times(A,+\infty)} \simeq \mathcal{L}_{2} \otimes \mathbf{k}_{(A,+\infty)}$ and the inclusion $\operatorname{Supp}\left(T_{c *} F_{1}\right) \subset M \times(c-A,+\infty)$, we get

$$
\begin{aligned}
R \operatorname{Hom}\left(F_{2}, T_{c *} F_{1}\right) & \simeq R \operatorname{Hom}\left(\mathcal{L}_{2} \otimes \mathbf{k}_{\mathbb{R}}, T_{c *} F_{1}\right) \\
& \simeq R \Gamma\left(M \times \mathbb{R} ; F_{1} \otimes\left(\mathcal{L}_{2}^{\otimes-1} \otimes \mathbf{k}_{\mathbb{R}}\right)\right)
\end{aligned}
$$

for any $c \geq c_{0}$. Since $\operatorname{SS}\left(F_{1} \otimes\left(\mathcal{L}_{2}^{\otimes-1} \otimes \mathbf{k}_{\mathbb{R}}\right)\right) \subset\{\tau \geq 0\}$, we can apply the microlocal Morse lemma (Proposition 2.3) and obtain

$$
\begin{aligned}
R \Gamma\left(M \times \mathbb{R} ; F_{1} \otimes\left(\mathcal{L}_{2}^{\otimes-1} \otimes \mathbf{k}_{\mathbb{R}}\right)\right) & \simeq R \Gamma\left(M \times(A,+\infty) ; F_{1} \otimes\left(\mathcal{L}_{2}^{\otimes-1} \otimes \mathbf{k}_{\mathbb{R}}\right)\right) \\
& \simeq R \Gamma\left(M \times(A,+\infty) ;\left(\mathcal{L}_{1} \otimes \mathcal{L}_{2}^{\otimes-1}\right) \otimes \mathbf{k}_{\mathbb{R}}\right) \\
& \simeq R \Gamma\left(M ; \mathcal{L}_{1} \otimes \mathcal{L}_{2}^{\otimes-1}\right) .
\end{aligned}
$$

The second assertion follows from Proposition 3.9.

Remark 4.3. In the special case where both $L_{1}$ and $L_{2}$ are the zero-section $T_{M}^{*} M$ of $T^{*} M$, (4.2) was already obtained by Guillermou-Schapira [GS14]. The outline of the proof is as follows. The simple sheaf quantization associated with the zero-section $T_{M}^{*} M$ and a rank 
1 locally constant sheaf $\mathcal{L} \in \operatorname{Mod}\left(\mathbf{k}_{M}\right)$ is isomorphic to $\mathcal{L} \otimes \mathbf{k}_{[0,+\infty)}$. In [GS14, Guillermou and Schapira proved that the functor

$$
\mathbf{D}^{\mathrm{b}}(M) \longrightarrow \mathcal{T}(M), F \longmapsto F \otimes \mathbf{k}_{[0,+\infty)}
$$

is fully faithful (see [GS14, Cor. 5.8]). We thus obtain

$$
\begin{aligned}
\operatorname{Hom}_{\mathcal{T}(M)}\left(\mathcal{L}_{2} \otimes \mathbf{k}_{[0,+\infty)}, \mathcal{L}_{1} \otimes \mathbf{k}_{[0,+\infty)}[k]\right) & \simeq \operatorname{Hom}_{\mathbf{D}^{\mathrm{b}}(M)}\left(\mathcal{L}_{2}, \mathcal{L}_{1}[k]\right) \\
& \simeq H^{k}\left(M ; \mathcal{L}_{1} \otimes \mathcal{L}_{2}^{\otimes-1}\right)
\end{aligned}
$$

for rank 1 locally constant sheaves $\mathcal{L}_{1}, \mathcal{L}_{2} \in \operatorname{Mod}\left(\mathbf{k}_{M}\right)$.

Moreover, we can prove (4.2) for general compact exact Lagrangians $L_{1}$ and $L_{2}$ using (4.7) and Proposition 4.4 below. The following was pointed out to the author by T. Kuwagaki.

Proposition 4.4. Let $L$ be a compact connected exact Lagrangian submanifold of $T^{*} M$. Let $\mathcal{L} \in \operatorname{Mod}\left(\mathbf{k}_{M}\right)$ be a locally constant sheaf of rank 1 and let $F \in \mathbf{D}_{\widehat{L} \cup T_{M \times \mathbb{R}}^{*}(M \times \mathbb{R}),+}^{\mathrm{b}}(M \times$ $\mathbb{R})$ be the simple sheaf quantization associated with $L$ satisfying $F_{+} \simeq \mathcal{L}$. Then

$$
F \simeq \mathcal{L} \otimes \mathbf{k}_{[0,+\infty)} \quad \text { in } \mathcal{T}(M)
$$

Proof. By the compactness of $L$, we can take a sufficiently large $A \in \mathbb{R}_{>0}$ such that $\widehat{L} \subset$ $T^{*}(M \times(-A, A))$. Since $\left.F\right|_{M \times(A,+\infty)} \simeq \mathcal{L} \otimes \mathbf{k}_{(A,+\infty)}$, there exists a canonical morphism

$$
F \longrightarrow \mathcal{L} \otimes \mathbf{k}_{[A+1,+\infty)} .
$$

The cone of this morphism is supported in $M \times[-A, A+1]$ and hence a torsion object. Therefore the morphism (4.9) is an isomorphism in $\mathcal{T}(M)$. A similar argument shows that the morphism $\mathcal{L} \otimes \mathbf{k}_{[0,+\infty)} \rightarrow \mathcal{L} \otimes \mathbf{k}_{[A+1,+\infty)}$ is an isomorphism in $\mathcal{T}(M)$.

By Theorem 3.11 and Proposition 4.2 we obtain the following:

Corollary 4.5. In the same notation as in Proposition 4.2, assume that $F_{i}$ is the canonical sheaf quantization of $\widehat{L}_{i}$, that is, $\mathcal{L}_{i} \simeq \mathbf{k}_{M}$ for $i=1,2$. Then

$$
\operatorname{Hom}_{\mathcal{T}(M)}\left(F_{2}, F_{1}[k]\right) \simeq H^{k}(M ; \mathbf{k}) \quad \text { for any } k \in \mathbb{Z} .
$$

In particular, $L_{1}$ and $L_{2}$ are mutually non-displaceable.

\subsection{Morse-Bott inequality for $\mathcal{H o m}^{\star}$}

In this subsection, we shall apply the Morse-Bott inequality for sheaves to $\mathcal{H o m}^{\star}\left(F_{2}, F_{1}\right)$. For this purpose, we estimate $\operatorname{SS}\left(\mathcal{H o m}{ }^{\star}\left(F_{2}, F_{1}\right)\right)$. Recall the isomorphism

$$
\mathcal{H o m}^{\star}\left(F_{2}, F_{1}\right) \simeq R s_{*} R \mathcal{H o m}\left(\tilde{q}_{2}^{-1} i^{-1} F_{2}, \tilde{q}_{1}^{!} F_{1}\right),
$$

where $\tilde{q}_{1}, \tilde{q}_{2}: M \times \mathbb{R} \times \mathbb{R} \rightarrow M \times \mathbb{R}$ are the projections, $s: M \times \mathbb{R} \times \mathbb{R} \rightarrow M \times \mathbb{R}$ is the addition map, and $i: M \times \mathbb{R} \rightarrow M \times \mathbb{R}$ is the involution $(x, t) \mapsto(x,-t)$. Since $\tilde{q}_{2}$ and $\tilde{q}_{1}$ are submersions, by Theorem 2.5(ii) we have inclusions

$$
\begin{aligned}
\operatorname{SS}\left(\tilde{q}_{2}^{-1} i^{-1} F_{2}\right) & \subset \tilde{q}_{2 d} \tilde{q}_{2 \pi}^{-1} \operatorname{SS}\left(i^{-1} F_{2}\right) \\
& =\left\{\begin{array}{l|l}
\left(x, t_{1}, t_{2} ; \tau_{2} \xi_{2}, 0,-\tau_{2}\right) & \begin{array}{l}
\tau_{2}>0,\left(x ; \xi_{2}\right) \in L_{2}, \\
t_{1} \in \mathbb{R}, t_{2}=f_{2}\left(x ; \xi_{2}\right)
\end{array}
\end{array}\right\}
\end{aligned}
$$


and

$$
\begin{aligned}
\operatorname{SS}\left(\tilde{q}_{1}^{!} F_{1}\right) & \subset \tilde{q}_{1 d} \tilde{q}_{1 \pi}^{-1} \operatorname{SS}\left(F_{1}\right) \\
& =\left\{\begin{array}{l|l}
\left(x, t_{1}, t_{2} ; \tau_{1} \xi_{1}, \tau_{1}, 0\right) & \begin{array}{l}
\tau_{1}>0,\left(x ; \xi_{1}\right) \in L_{1}, \\
t_{1}=-f_{1}\left(x ; \xi_{1}\right), t_{2} \in \mathbb{R}
\end{array}
\end{array}\right\} .
\end{aligned}
$$

Hence $\operatorname{SS}\left(\tilde{q}_{2}^{-1} i^{-1} F_{2}\right) \cap \operatorname{SS}^{\circ}\left(\tilde{q}_{1}^{!} F_{1}\right)=\emptyset$, and by Proposition 2.7 we obtain

$$
\begin{aligned}
& \operatorname{SS}\left(R \mathcal{H} \operatorname{om}\left(\tilde{q}_{2}^{-1} i^{-1} F_{2}, \tilde{q}_{1}^{!} F_{1}\right)\right) \subset \operatorname{SS}\left(\tilde{q}_{2}^{-1} i^{-1} F_{2}\right)^{a}+\operatorname{SS}\left(\tilde{q}_{1}^{!} F_{1}\right) \\
& =\left\{\begin{array}{l|l}
\left(x, t_{1}, t_{2} ; \tau_{1} \xi_{1}-\tau_{2} \xi_{2}, \tau_{1}, \tau_{2}\right) & \begin{array}{l}
\tau_{1}, \tau_{2}>0, \\
\left(x ; \xi_{1}\right) \in L_{1},\left(x ; \xi_{2}\right) \in L_{2}, \\
t_{1}=-f_{1}\left(x ; \xi_{1}\right), t_{2}=f_{2}\left(x ; \xi_{2}\right)
\end{array}
\end{array}\right\} \\
& =: \Lambda_{M \times \mathbb{R} \times \mathbb{R} .}
\end{aligned}
$$

Lemma 4.6. One has

$$
\begin{aligned}
& v_{d}^{-1}\left(\overline{v_{\pi}\left(\Lambda_{M \times \mathbb{R} \times \mathbb{R}} \cup T_{M \times \mathbb{R} \times \mathbb{R}}^{*}(M \times \mathbb{R} \times \mathbb{R})\right)}\right) \\
= & v_{d}^{-1} v_{\pi}\left(\Lambda_{M \times \mathbb{R} \times \mathbb{R}} \cup T_{M \times \mathbb{R} \times \mathbb{R}}^{*}(M \times \mathbb{R} \times \mathbb{R})\right) \\
= & s_{\pi} s_{d}^{-1}\left(\Lambda_{M \times \mathbb{R} \times \mathbb{R}} \cup T_{M \times \mathbb{R} \times \mathbb{R}}^{*}(M \times \mathbb{R} \times \mathbb{R})\right) .
\end{aligned}
$$

In other words,

$$
\begin{aligned}
& s_{\sharp}\left(\Lambda_{M \times \mathbb{R} \times \mathbb{R}} \cup T_{M \times \mathbb{R} \times \mathbb{R}}^{*}(M \times \mathbb{R} \times \mathbb{R})\right) \\
= & s_{\pi} s_{d}^{-1}\left(\Lambda_{M \times \mathbb{R} \times \mathbb{R}} \cup T_{M \times \mathbb{R} \times \mathbb{R}}^{*}(M \times \mathbb{R} \times \mathbb{R})\right) .
\end{aligned}
$$

See Subsection 2.4 for the notation $v_{\pi}, v_{d}$, and $s_{\sharp}$ associated with the constant linear $\operatorname{map} s: M \times \mathbb{R} \times \mathbb{R} \rightarrow M \times \mathbb{R}$.

Proof. Define $\Lambda^{\prime} \subset T^{*} M \times \mathbb{R} \times(\mathbb{R} \times \mathbb{R})$ by

$$
\Lambda^{\prime}:=\left\{\begin{array}{l|l}
\left(\left(x ; \tau_{1} \xi_{1}-\tau_{2} \xi_{2}\right),\left(t ; \tau_{1}, \tau_{2}\right)\right) & \begin{array}{l}
\tau_{1}, \tau_{2}>0,\left(x ; \xi_{1}\right) \in L_{1},\left(x ; \xi_{2}\right) \in L_{2}, \\
t=f_{2}\left(x ; \xi_{2}\right)-f_{1}\left(x ; \xi_{1}\right)
\end{array}
\end{array}\right\} .
$$

Then the set $v_{\pi}\left(\Lambda_{M \times \mathbb{R} \times \mathbb{R}} \cup T_{M \times \mathbb{R} \times \mathbb{R}}^{*}(M \times \mathbb{R} \times \mathbb{R})\right)$ is equal to $\Lambda^{\prime} \cup\left(T_{M}^{*} M \times \mathbb{R} \times\{(0,0)\}\right) \subset$ $T^{*} M \times \mathbb{R} \times(\mathbb{R} \times \mathbb{R})$. It suffices to check that $\Lambda^{\prime} \cup\left(T_{M}^{*} M \times \mathbb{R} \times\{(0,0)\}\right)$ is equal to its closure. By the compactness of $L_{1}$ and $L_{2}$, there exists $C \in \mathbb{R}_{>0}$ such that $|\xi| \leq C\left(\left|\tau_{1}\right|+\left|\tau_{2}\right|\right)$ for any $\left((x ; \xi),\left(t ; \tau_{1}, \tau_{2}\right)\right) \in \Lambda^{\prime}$. Therefore the same inequality holds on the closure $\overline{\Lambda^{\prime}}$ of $\Lambda^{\prime}$. Hence if $\left((x ; \xi),\left(t ; \tau_{1}, \tau_{2}\right)\right) \in \overline{\Lambda^{\prime}}$ and $\tau_{1}=\tau_{2}=0$ then $\xi=0$, which proves the equality.

By Proposition 2.9, Lemma 4.6, and (4.14), $\operatorname{SS}^{\circ}\left(\mathcal{H o m}^{\star}\left(F_{2}, F_{1}\right)\right)$ is estimated as

$$
\begin{aligned}
& \operatorname{SSS}\left(\mathcal{H o m}{ }^{\star}\left(F_{2}, F_{1}\right)\right) \subset s_{\sharp}\left(\Lambda_{M \times \mathbb{R} \times \mathbb{R}} \cup T_{M \times \mathbb{R} \times \mathbb{R}}^{*}(M \times \mathbb{R} \times \mathbb{R})\right) \cap \stackrel{\circ}{T}^{*}(M \times \mathbb{R}) \\
& =s_{\pi} s_{d}^{-1}\left(\Lambda_{M \times \mathbb{R} \times \mathbb{R}} \cup T_{M \times \mathbb{R} \times \mathbb{R}}^{*}(M \times \mathbb{R} \times \mathbb{R})\right) \cap \stackrel{\circ}{T}^{*}(M \times \mathbb{R}) \\
& \subset\left\{\begin{array}{l|l}
\left(x, t ; \tau\left(\xi_{1}-\xi_{2}\right), \tau\right) & \begin{array}{l}
\tau>0, \\
\left(x ; \xi_{1}\right) \in L_{1},\left(x ; \xi_{2}\right) \in L_{2} \\
t=f_{2}\left(x ; \xi_{2}\right)-f_{1}\left(x ; \xi_{1}\right)
\end{array}
\end{array}\right\} \\
& =: \Lambda_{M \times \mathbb{R}} .
\end{aligned}
$$


Let $t: M \rightarrow \mathbb{R}$ be the function $(x, t) \mapsto t$. Then, by (4.18), we obtain

$$
\Gamma_{d t} \cap \operatorname{SS}\left(\mathcal{H o m}^{\star}\left(F_{2}, F_{1}\right)\right) \subset\left\{\begin{array}{l|l}
(x, t ; 0,1) & \begin{array}{l}
\exists(x ; \xi) \in L_{1} \cap L_{2}, \\
t=f_{2}(x ; \xi)-f_{1}(x ; \xi)
\end{array}
\end{array}\right\} .
$$

By this inclusion, we find that $\left.R \Gamma_{M \times[c,+\infty)}\left(\mathcal{H o m}{ }^{\star}\left(F_{2}, F_{1}\right)\right)\right|_{M \times\{c\}} \simeq 0$ if $c \notin\left\{f_{2}(p)-f_{1}(p) \mid\right.$ $\left.p \in L_{1} \cap L_{2}\right\}$.

Proposition 4.7. Let $a, b \in \mathbb{R}$ with $a<b$ or $a \in \mathbb{R}, b=+\infty$. Assume that

(1) the point $a \in \mathbb{R}$ is not an accumulation point of $\left\{f_{2}(p)-f_{1}(p) \mid p \in L_{1} \cap L_{2}\right\} \subset \mathbb{R}$,

(2) the set $\left\{f_{2}(p)-f_{1}(p) \mid p \in L_{1} \cap L_{2}\right\} \cap[a, b)$ is finite,

(3) the object $R \Gamma\left(M \times\{c\} ;\left.R \Gamma_{M \times[c,+\infty)}\left(\mathcal{H o m}^{\star}\left(F_{2}, F_{1}\right)\right)\right|_{M \times\{c\}}\right)$ has finite-dimensional cohomology for any $a \leq c<b$.

Then

$$
\begin{gathered}
\sum_{a \leq c<b} \operatorname{dim} H^{k} R \Gamma\left(M \times\{c\} ;\left.R \Gamma_{M \times[c,+\infty)}\left(\mathcal{H o m}^{\star}\left(F_{2}, F_{1}\right)\right)\right|_{M \times\{c\}}\right) \\
\geq \operatorname{dim} H^{k} R \Gamma_{M \times[a, b)}\left(M \times(-\infty, b) ; \mathcal{H o m}{ }^{\star}\left(F_{2}, F_{1}\right)\right)
\end{gathered}
$$

for any $k \in \mathbb{Z}$.

Proof. We set $H:=\mathcal{H o m}^{\star}\left(F_{2}, F_{1}\right)$. By assumption (1) we can take $a^{\prime}<a$ such that

$$
f_{1}(p)-f_{2}(p) \notin\left[a^{\prime}, a\right) \quad \text { for any } p \in L_{1} \cap L_{2} .
$$

By (4.18) and (4.21) we have $\mathrm{S} S(H) \cap \mathrm{SS}\left(\mathbf{k}_{M \times\left[a^{\prime},+\infty\right)}\right)=\emptyset$. Hence by Proposition 2.7] we obtain

$$
\begin{aligned}
\operatorname{SiS}\left(R \Gamma_{M \times\left[a^{\prime},+\infty\right)}(H)\right) & =\operatorname{SS}\left(R \mathcal{H o m}\left(\mathbf{k}_{M \times\left[a^{\prime},+\infty\right)}, H\right)\right) \\
& \subset \Lambda_{M \times \mathbb{R}} \cap \pi^{-1}\left(\left\{t>a^{\prime}\right\}\right)+\left\{\left(x, a^{\prime} ; 0,-\tau^{\prime}\right) \mid \tau^{\prime}>0\right\} .
\end{aligned}
$$

Set $H^{\prime}:=\left.R \Gamma_{M \times\left[a^{\prime},+\infty\right)}(H)\right|_{M \times(-\infty, b)} \in \mathbf{D}^{\mathrm{b}}(M \times(-\infty, b))$ and let $t: M \times(-\infty, b) \rightarrow$ $\mathbb{R}$ be the function $(x, t) \mapsto t$. We shall apply the Morse-Bott inequality for sheaves (Theorem 2.10) to $H^{\prime}$ and $t: M \times(-\infty, b) \rightarrow \mathbb{R}$. Combining (4.18) with (4.22), we get

$$
\Gamma_{d t} \cap \operatorname{SS}\left(H^{\prime}\right) \subset\left\{(x, t ; 0,1) \mid \exists p \in L_{1} \cap L_{2}, x=\pi(p), a^{\prime}<t=f_{2}(p)-f_{1}(p)<b\right\} .
$$

Hence the conditions in Theorem 2.10 are satisfied by (4.21) and assumptions (2) and (3). Hence we have the inequality

$$
\begin{gathered}
\sum_{a^{\prime}<c<b} \operatorname{dim} H^{k} R \Gamma\left(M \times\{c\} ;\left.R \Gamma_{M \times[c,+\infty)}(H)\right|_{M \times\{c\}}\right) \\
\geq \operatorname{dim} H^{k} R \Gamma_{M \times\left[a^{\prime}, b\right)}(M \times(-\infty, b) ; H)
\end{gathered}
$$

for any $k \in \mathbb{Z}$. Moreover, by (4.18), (4.21), and (4.22), we get $\Gamma_{d t} \cap \operatorname{SS}\left(H^{\prime}\right) \cap \pi^{-1}(M \times$ $\left.\left[a^{\prime}, a\right)\right)=\emptyset$. Applying the microlocal Morse lemma (Proposition 2.3), we have

$$
\begin{aligned}
R \Gamma_{M \times\left[a^{\prime}, a\right)}(M \times(-\infty, a) ; H) & \simeq R \Gamma\left(M \times(-\infty, a) ; H^{\prime}\right) \\
& \simeq R \Gamma\left(\left(-\infty, a^{\prime}\right) ; H^{\prime}\right) \simeq 0 .
\end{aligned}
$$

Thus we get $R \Gamma_{M \times[a, b)}(M \times(-\infty, b) ; H) \simeq R \Gamma_{M \times\left[a^{\prime}, b\right)}(M \times(-\infty, b) ; H)$. On the other hand, by (4.21), $\left.R \Gamma_{M \times[c,+\infty)}(H)\right|_{M \times\{c\}} \simeq 0$ for $c \in\left[a^{\prime}, a\right)$ and the left-hand side of (4.24) is equal to that of (4.20). This completes the proof. 
Remark 4.8. C. Viterbo announced that he had found some relation between the section of $\mathcal{H o m}^{\star}\left(F_{2}, F_{1}\right)$ on $M \times(-\infty, \lambda)$ and the Floer cohomology complex $\mathrm{CF}_{<\lambda}\left(L_{2}, L_{1}\right)$ filtered by $\left\{p \in L_{1} \cap L_{2} \mid f_{2}(p)-f_{1}(p)<\lambda\right\}$. Inspired by his work, in Proposition 4.7 we consider not only the section on $M \times \mathbb{R}$ but also that on $M \times(-\infty, b)$.

\subsection{Microlocalization of $\mathcal{H o m}^{\star}$}

In this subsection we describe $R \Gamma\left(M \times\{c\} ;\left.R \Gamma_{M \times[c,+\infty)}\left(\mathcal{H o m}^{\star}\left(F_{2}, F_{1}\right)\right)\right|_{M \times\{c\}}\right)$ in terms of the functor $\mu$ hom. Applying $T_{c *}$ to $F_{2}$, we may assume $c=0$. The following lemma follows from Proposition 2.16,

Lemma 4.9. Set $V_{+}:=\{(x, 0 ; 0, \tau) \mid \tau>0\} \subset T_{M \times\{0\}}^{*}(M \times \mathbb{R})$. Then

$$
\begin{gathered}
R \Gamma\left(M \times\{0\} ;\left.R \Gamma_{M \times[0,+\infty)}\left(\mathcal{H o m}^{\star}\left(F_{2}, F_{1}\right)\right)\right|_{M \times\{0\}}\right) \\
\simeq R \Gamma\left(V_{+} ;\left.\mu_{M \times\{0\}}\left(\mathcal{H o m}{ }^{\star}\left(F_{2}, F_{1}\right)\right)\right|_{V_{+}}\right) .
\end{gathered}
$$

Recall the isomorphism

$$
\mathcal{H o m}^{\star}\left(F_{2}, F_{1}\right) \simeq R s_{*} \delta^{!} R \mathcal{H} \text { om }\left(q_{2}^{-1} i^{-1} F_{2}, q_{1}^{!} F_{1}\right),
$$

where $s: M \times \mathbb{R} \times \mathbb{R} \rightarrow M \times \mathbb{R}$ is the addition map, $\delta: M \times \mathbb{R} \times \mathbb{R} \rightarrow M \times M \times \mathbb{R} \times \mathbb{R}$ is the diagonal embedding, and $q_{i}: M \times \mathbb{R} \times M \times \mathbb{R} \rightarrow M \times \mathbb{R}$ is the $i$ th projection. The morphism $s$ induces the following commutative diagram, where we omit $T^{*} M$ (resp. $T_{M}^{*} M$ ) in the first (resp. second) row and use the same symbol $s$ for the addition map $\mathbb{R} \times \mathbb{R} \rightarrow \mathbb{R}$ :

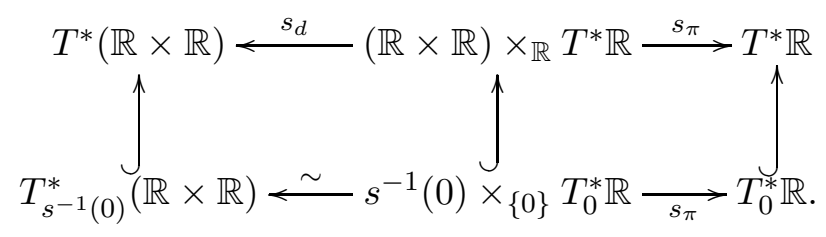

We denote by $\pi_{s}: T_{M}^{*} M \times T_{s^{-1}(0)}^{*}(\mathbb{R} \times \mathbb{R}) \rightarrow T_{M}^{*} M \times T_{0}^{*} \mathbb{R} \simeq T_{M \times\{0\}}^{*}(M \times \mathbb{R})$ the induced morphism in the second row in the above diagram. On the other hand, the morphism $\delta$ induces the following commutative diagram, where we omit $T_{s^{-1}(0)}^{*}(\mathbb{R} \times \mathbb{R})$ :

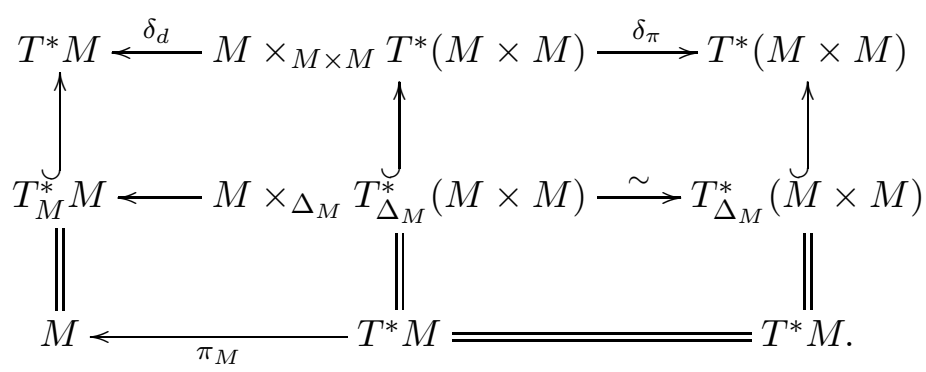

Moreover, let $\iota: T^{*} \mathbb{R} \simeq T_{\Delta_{\mathbb{R}}}^{*}(\mathbb{R} \times \mathbb{R}) \stackrel{\sim}{\rightarrow} T_{s^{-1}(0)}^{*}(\mathbb{R} \times \mathbb{R})$ be the isomorphism of line bundles defined by $\left(t_{1}, t_{2}, \tau,-\tau\right) \mapsto\left(t_{1},-t_{2}, \tau, \tau\right)$. We also use the same symbol $\iota$ for the induced isomorphism $T^{*}(M \times \mathbb{R}) \simeq T^{*} M \times T_{\Delta_{\mathbb{R}}}^{*}(\mathbb{R} \times \mathbb{R}) \stackrel{\sim}{\rightarrow} T^{*} M \times T_{s^{-1}(0)}^{*}(\mathbb{R} \times \mathbb{R})$.

Proposition 4.10. Set $V_{+}:=\{(x, 0 ; 0, \tau) \mid \tau>0\} \subset T_{M \times\{0\}}^{*}(M \times \mathbb{R})$ as in Lemma 4.9 and define $\pi^{\prime}:=\pi_{s} \circ \pi_{M} \circ \iota: T^{*}(M \times \mathbb{R}) \rightarrow T_{M \times\{0\}}^{*}(M \times \mathbb{R})$. Then

$$
\left.\left.\mu_{M \times\{0\}}\left(\mathcal{H o m}^{\star}\left(F_{2}, F_{1}\right)\right)\right|_{V_{+}} \simeq\left(R \pi_{*}^{\prime} \mu h o m\left(F_{2}, F_{1}\right)\right)\right|_{V_{+}} .
$$


Proof. (a) Set $H=\mathcal{H o m}^{\star}\left(F_{2}, F_{1}\right)$. First, we note that $\mu_{M \times\{0\}}(H) \simeq \mu_{M \times\{0\}}\left(\left.H\right|_{M \times(-1,1)}\right)$. Set $U:=M \times(-1,1) \subset M \times \mathbb{R}$. There exists a sufficiently large $A \in \mathbb{R}_{>0}$ such that $F_{1}$ and $F_{2}$ are locally constant on $M \times(A-2,+\infty)$. Since the problem is local on $M$, we may assume that $F_{1}$ and $F_{2}$ are constant on $M \times(A-2,+\infty)$ from the beginning. Then $\tilde{q}_{1}^{!} F_{1} \simeq \tilde{q}_{1}^{-1} F_{1}[1]$ is constant on $s^{-1}(U) \cap(M \times \mathbb{R} \times(-\infty,-A+1))$, which implies isomorphisms

$$
\begin{aligned}
& \left.R \mathcal{H o m}\left(\tilde{q}_{2}^{-1} i^{-1} \mathbf{k}_{M \times[A,+\infty)}, \tilde{q}_{1}^{!} F_{1}\right)\right|_{s^{-1}(U)} \\
\simeq & \left.R \mathcal{H o m}\left(\mathbf{k}_{M \times \mathbb{R} \times(-\infty,-A]}, \mathbf{k}_{M \times \mathbb{R} \times \mathbb{R}}[1]\right)\right|_{s^{-1}(U)} \\
\simeq & R \Gamma_{s^{-1}(U) \cap(M \times \mathbb{R} \times(-\infty,-A])}\left(\mathbf{k}_{s^{-1}(U)}\right)[1] .
\end{aligned}
$$

Therefore we obtain

$$
\left.\left(R s_{*} R \mathcal{H o m}\left(\tilde{q}_{2}^{-1} i^{-1} \mathbf{k}_{M \times[A,+\infty)}, \tilde{q}_{1}^{!} F_{1}\right)\right)\right|_{U} \simeq 0 .
$$

By the distinguished triangle

$$
F_{2}^{\prime} \longrightarrow F_{2} \longrightarrow \mathbf{k}_{M \times[A,+\infty)} \stackrel{+1}{\longrightarrow}
$$

with $F_{2}^{\prime}$ supported in some compact subset, we find that

$$
\left.\left.\left(R s_{*} R \mathcal{H o m}\left(\tilde{q}_{2}^{-1} i^{-1} F_{2}, \tilde{q}_{1}^{!} F_{1}\right)\right)\right|_{U} \simeq\left(R s_{*} R \mathcal{H o m}\left(\tilde{q}_{2}^{-1} i^{-1} F_{2}^{\prime}, \tilde{q}_{1}^{!} F_{1}\right)\right)\right|_{U}
$$

and $s$ is proper on $\operatorname{Supp}\left(R \mathcal{H} o m\left(\tilde{q}_{2}^{-1} i^{-1} F_{2}^{\prime}, \tilde{q}_{1}^{!} F_{1}\right)\right)$.

(b) Since $s$ is proper on the support, by Proposition 2.12(i), we have

$$
\mu_{M \times\{0\}}\left(R s_{*} R \mathcal{H} \operatorname{om}\left(\tilde{q}_{2}^{-1} i^{-1} F_{2}^{\prime}, \tilde{q}_{1}^{!} F_{1}\right)\right) \simeq R \pi_{s *} \mu_{M \times s^{-1}(0)}\left(R \mathcal{H o m}\left(\tilde{q}_{2}^{-1} i^{-1} F_{2}^{\prime}, \tilde{q}_{1}^{!} F_{1}\right)\right) .
$$

Moreover, since $\delta$ is non-characteristic for $\operatorname{SS}\left(R \mathcal{H o m}\left(\tilde{q}_{2}^{-1} i^{-1} F_{2}^{\prime}, \tilde{q}_{1}^{!} F_{1}\right)\right)$ and $\left.\delta\right|_{M \times s^{-1}(0)}: M \times$ $s^{-1}(0) \rightarrow \Delta_{M} \times s^{-1}(0)$ is a submersion, by Proposition 2.12(ii) we obtain

$$
\begin{aligned}
\mu_{M \times s^{-1}(0)}\left(R \mathcal{H o m}\left(\tilde{q}_{2}^{-1} i^{-1} F_{2}^{\prime}, \tilde{q}_{1}^{!} F_{1}\right)\right) & \simeq \mu_{M \times s^{-1}(0)}\left(\delta^{!} R \mathcal{H o m}\left(q_{2}^{-1} i^{-1} F_{2}^{\prime}, q_{1}^{!} F_{1}\right)\right) \\
& \simeq R \pi_{M *} \mu_{\Delta_{M} \times s^{-1}(0)} R \mathcal{H o m}\left(q_{2}^{-1} i^{-1} F_{2}^{\prime}, q_{1}^{!} F_{1}\right) .
\end{aligned}
$$

Let $i_{2}: M \times \mathbb{R} \times \mathbb{R} \rightarrow M \times \mathbb{R} \times \mathbb{R}$ be the involution $\left(x, t_{1}, t_{2}\right) \mapsto\left(x, t_{1},-t_{2}\right)$. Note that the associated automorphism of $T^{*} M \times T^{*}(\mathbb{R} \times \mathbb{R})$ induces $\iota: T^{*} M \times T_{\Delta_{\mathbb{R}}}^{*}(\mathbb{R} \times \mathbb{R}) \stackrel{\sim}{\rightarrow}$ $T^{*} M \times T_{s^{-1}(0)}^{*}(\mathbb{R} \times \mathbb{R})$. Then, by Proposition $2.12(\mathrm{i})$ again, we have

$$
\begin{aligned}
\mu_{\Delta_{M} \times s^{-1}(0)} R \mathcal{H o m}\left(i_{2}^{-1} q_{2}^{-1} F_{2}^{\prime}, q_{1}^{!} F_{1}\right) & \simeq \mu_{\Delta_{M} \times s^{-1}(0)} i_{2 *} R \mathcal{H o m}\left(q_{2}^{-1} F_{2}^{\prime}, q_{1}^{!} F_{1}\right) \\
& \simeq \iota_{*} \mu_{\Delta_{M \times \mathbb{R}}} R \mathcal{H o m}\left(q_{2}^{-1} F_{2}^{\prime}, q_{1}^{!} F_{1}\right) \\
& \simeq \iota_{*} \mu h o m\left(F_{2}^{\prime}, F_{1}\right) .
\end{aligned}
$$

(c) By Proposition 2.15 we have

$$
\operatorname{Supp}\left(\mu h o m\left(\mathbf{k}_{M \times[A,+\infty)}, F_{1}\right)\right) \subset T_{M \times \mathbb{R}}^{*}(M \times \mathbb{R}) .
$$

Thus, by the distinguished triangle (4.33), we get

$$
\left.\left.\operatorname{\mu hom}\left(F_{2}^{\prime}, F_{1}\right)\right|_{\{\tau>0\}} \stackrel{\sim}{\rightarrow} \mu h o m\left(F_{2}, F_{1}\right)\right|_{\{\tau>0\}},
$$

which completes the proof. 
We define an open subset $\Omega_{+}$of $T^{*}(M \times \mathbb{R}) \simeq T^{*} M \times T^{*} \mathbb{R}$ by $\Omega_{+}:=\{\tau>0\} \subset$ $T^{*}(M \times \mathbb{R})$. Combining Proposition 4.7 with Lemma 4.9 and Proposition 4.10, we obtain the following:

Proposition 4.11. Let $a, b \in \mathbb{R}$ with $a<b$ or $a \in \mathbb{R}, b=+\infty$. Assume

(1) the point $a \in \mathbb{R}$ is not an accumulation point of $\left\{f_{2}(p)-f_{1}(p) \mid p \in L_{1} \cap L_{2}\right\} \subset \mathbb{R}$,

(2) the set $\left\{f_{2}(p)-f_{1}(p) \mid p \in L_{1} \cap L_{2}\right\} \cap[a, b) \subset \mathbb{R}$ is finite,

(3) the object $R \Gamma\left(\Omega_{+} ;\left.\mu h o m\left(T_{c *} F_{2}, F_{1}\right)\right|_{\Omega_{+}}\right)$has finite-dimensional cohomology for any $a \leq c<b$.

Then

$$
\begin{aligned}
& \sum_{a \leq c<b} \operatorname{dim} H^{k} R \Gamma\left(\Omega_{+} ;\left.\mu h o m\left(T_{c *} F_{2}, F_{1}\right)\right|_{\Omega_{+}}\right) \\
& \quad \geq \operatorname{dim} H^{k} R \Gamma_{M \times[a, b)}\left(M \times(-\infty, b) ; \mathcal{H o m}{ }^{\star}\left(F_{2}, F_{1}\right)\right)
\end{aligned}
$$

for any $k \in \mathbb{Z}$.

\subsection{Clean intersections of compact exact Lagrangian submanifolds}

Throughout this subsection we assume the following:

Assumption 4.12. The Lagrangian submanifolds $L_{1}$ and $L_{2}$ intersect cleanly, that is, $L_{1} \cap L_{2}$ is a submanifold of $T^{*} M$ and $T_{p}\left(L_{1} \cap L_{2}\right)=T_{p} L_{1} \cap T_{p} L_{2}$ for any $p \in L_{1} \cap L_{2}$.

Under the assumption, the intersection $L_{1} \cap L_{2}$ has finitely many connected components, which are compact submanifolds of $T^{*} M$, and the value $f_{2}(p)-f_{1}(p)$ is constant on each component. In particular, the set $\left\{f_{2}(p)-f_{1}(p) \mid p \in L_{1} \cap L_{2}\right\} \subset \mathbb{R}$ is finite. For a component $C$ of $L_{1} \cap L_{2}$, we define $f_{21}(C):=f_{2}(p)-f_{1}(p)$, taking some $p \in C$.

Under Assumption 4.12, we shall compute $\left.\mu h o m\left(T_{c *} F_{2}, F_{1}\right)\right|_{\Omega_{+}}$. Again, we may assume $c=0$. Recall that we have set $\Lambda_{i}:=\widehat{L_{i}}$ for simplicity of notation. The following lemma is obtained in Gui12, Lem. 6.14].

Lemma 4.13. Under Assumption 4.12, $\mu$ hom $\left.\left(F_{2}, F_{1}\right)\right|_{\Omega_{+}}$is supported in $\Lambda_{1} \cap \Lambda_{2}$ and has locally constant cohomology sheaves.

Proof. For completeness, we also give a proof here. By Proposition 2.15, we have

$$
\begin{aligned}
\operatorname{Supp}\left(\left.\mu \operatorname{hom}\left(F_{2}, F_{1}\right)\right|_{\Omega_{+}}\right) & \subset \Lambda_{1} \cap \Lambda_{2}, \\
\operatorname{SS}\left(\left.\mu \operatorname{hom}\left(F_{2}, F_{1}\right)\right|_{\Omega_{+}}\right) & \subset-\mathbf{h}^{-1}\left(C\left(\Lambda_{1}, \Lambda_{2}\right)\right) \cap T^{*} \Omega_{+} .
\end{aligned}
$$

Set $\Lambda_{12}:=\Lambda_{1} \cap \Lambda_{2}$. Since $\Lambda_{1}$ and $\Lambda_{2}$ intersect cleanly, we have

$$
C\left(\Lambda_{1}, \Lambda_{2}\right)=\left.T \Lambda_{1}\right|_{\Lambda_{12}}+\left.T \Lambda_{2}\right|_{\Lambda_{12}} .
$$

Since $\Lambda_{i}$ is Lagrangian, we get $-\mathbf{h}^{-1}\left(T \Lambda_{i}\right) \subset T_{\Lambda_{i}}^{*} T^{*}(M \times \mathbb{R})$ for $i=1,2$. In particular, $-\mathbf{h}^{-1}\left(\left.T \Lambda_{i}\right|_{\Lambda_{12}}\right) \subset T_{\Lambda_{12}}^{*} T^{*}(M \times \mathbb{R})$. Hence we obtain

$$
-\mathbf{h}^{-1}\left(C\left(\Lambda_{1}, \Lambda_{2}\right)\right) \cap T^{*} \Omega_{+} \subset T_{\Lambda_{12}}^{*} T^{*}(M \times \mathbb{R}) .
$$

Hence by (4.41), $\operatorname{SS}\left(\left.\mu h o m\left(F_{2}, F_{1}\right)\right|_{\Omega_{+}}\right) \subset T_{\Lambda_{12}}^{*} T^{*}(M \times \mathbb{R})$, which proves the result. 
Let $C_{1}, \ldots, C_{n_{0}}$ be the connected components of $L_{1} \cap L_{2}$ with $f_{21}\left(C_{j}\right)=0(j=$ $\left.1, \ldots, n_{0}\right)$. For a component $C_{j}$, we define a closed subset $\widehat{C_{j}}$ of $\Omega_{+} \subset T^{*}(M \times \mathbb{R})$ by

$$
\widehat{C_{j}}:=\left\{(x, t ; \xi, \tau) \mid \tau>0,(x ; \xi / \tau) \in C_{j}, t=-f_{1}(x ; \xi / \tau)\left(=-f_{2}(x ; \xi / \tau)\right)\right\} .
$$

Note that $\widehat{C_{j}} / \mathbb{R}_{>0} \simeq C_{j}$. We also denote by $d_{i}: \Lambda_{i} \rightarrow \frac{1}{2} \mathbb{Z}$ the function which assigns the shift of $F_{i}$. Since the function $d_{i}$ is invariant under the $\mathbb{R}_{>0}$-action, we use the same symbol $d_{i}$ for the function $L_{i}=\Lambda_{i} / \mathbb{R}_{>0} \rightarrow \frac{1}{2} \mathbb{Z}$ (see also Section Cl).

Theorem 4.14. Under Assumption 4.12 and in the notation above, assume, moreover, that $\mathbf{k}=\mathbb{F}_{2}=\mathbb{Z} / 2 \mathbb{Z}$. Then

$$
\left.\mu h o m\left(F_{2}, F_{1}\right)\right|_{\Omega_{+}} \simeq \bigoplus_{j=1}^{n_{0}} \mathbf{k}_{\widehat{C_{j}}}\left[-s\left(C_{j}\right)\right]
$$

where $s\left(C_{j}\right) \in \mathbb{Z}$ is given by

$$
s\left(C_{j}\right):=d_{2}(p)-d_{1}(p)+\frac{1}{2}\left(\operatorname{dim} M-\operatorname{dim} C_{j}\right)-\frac{1}{2} \tau\left(T_{p} L_{2}, T_{p} L_{1}, T_{p}\left(T_{\pi(p)}^{*} M\right)\right)
$$

with $p \in C_{j}$. In particular,

$$
R \Gamma\left(\Omega_{+} ;\left.\mu h o m\left(F_{2}, F_{1}\right)\right|_{\Omega_{+}}\right) \simeq \bigoplus_{j=1}^{n_{0}} R \Gamma\left(C_{j} ; \mathbf{k}_{C_{j}}\right)\left[-s\left(C_{j}\right)\right] .
$$

Proof. (a) By Lemma 4.13, $\mu$ hom $\left.\left(F_{2}, F_{1}\right)\right|_{\Lambda_{1} \cap \Lambda_{2}}$ has locally constant cohomology sheaves. Fix $p \in C_{j}$ and let us compute the stalk at $p^{\prime}:=(p, 0 ; 1) \in \widehat{C_{j}}$. There exists a Hamiltonian diffeomorphism with compact support $\psi: T^{*} M \rightarrow T^{*} M$ such that $\psi\left(L_{i}\right)$ is a graph $\Gamma_{d \varphi_{i}}$ of a $C^{\infty}$-function $\varphi_{i}: M \rightarrow \mathbb{R}$ in a neighborhood of $\psi(p)$ for $i=1,2$. Let $\widehat{\psi}: \stackrel{\circ}{*}^{*}(M \times$ $\mathbb{R}) \rightarrow T^{*}(M \times \mathbb{R})$ be the homogeneous Hamiltonian diffeomorphism associated with $\psi$ and $K \in \mathbf{D}^{\mathrm{b}}(M \times \mathbb{R} \times M \times \mathbb{R})$ be the sheaf quantization of $\widehat{\psi}$. For simplicity of notation we set $\chi=\widehat{\psi}$. By Proposition 2.20, in a neighborhood of $\chi\left(p^{\prime}\right)$, we have the isomorphism

$$
\mu h o m\left(K \circ F_{2}, K \circ F_{1}\right) \simeq \chi_{*} \mu h o m\left(F_{2}, F_{1}\right) .
$$

Moreover, by Proposition 2.21, $K \circ F_{i}$ is simple with shift $d_{i}(p)+d^{\prime}-\delta_{i}$ along $\chi\left(\Lambda_{i}\right)$ at $\chi\left(p^{\prime}\right)$, where $d^{\prime}$ is the shift of $K$ at $\left(\chi\left(p^{\prime}\right), p^{a}\right)$ and

$$
\delta_{i}:=\frac{1}{2}(\operatorname{dim} M+1)+\frac{1}{2} \tau\left(\lambda_{\infty}\left(p^{\prime}\right), \lambda_{\Lambda_{i}}\left(p^{\prime}\right), \chi^{-1}\left(\lambda_{\infty}\left(\chi\left(p^{\prime}\right)\right)\right)\right) .
$$

Here we use the symbols $\lambda_{\Lambda}(p)$ and $\lambda_{\infty}(p)$ defined in (2.31). Hence we obtain the isomorphism $K \circ F_{i} \simeq \mathbf{k}_{N_{i}}\left[d_{i}(p)+d^{\prime}-\delta_{i}-\frac{1}{2}\right]$ in $\mathbf{D}^{\mathrm{b}}\left(M \times \mathbb{R} ; \chi\left(p^{\prime}\right)\right)$, where $N_{i}:=\{(x, t) \in$ $\left.M \times \mathbb{R} \mid \varphi_{i}(x)+t=0\right\}$ (see also Example 2.19). Thus we get

$$
\begin{aligned}
\operatorname{\mu hom}\left(F_{2}, F_{1}\right)_{p^{\prime}} & \simeq \mu h o m\left(\mathbf{k}_{N_{2}}, \mathbf{k}_{N_{1}}\right)_{\chi\left(p^{\prime}\right)}\left[d_{1}(p)-d_{2}(p)-\delta_{1}+\delta_{2}\right] \\
& \simeq \mu_{N_{2}}\left(\mathbf{k}_{N_{1}}\right)_{\chi\left(p^{\prime}\right)}\left[d_{1}(p)-d_{2}(p)-\delta_{1}+\delta_{2}\right]
\end{aligned}
$$

where we used Proposition 2.14(iii) for the second isomorphism. We introduce a new local coordinate system $\left(x, t^{\prime}\right)$ on $M \times \mathbb{R}$ by $t^{\prime}:=t+\varphi_{2}(x)$. Then $N_{2}=\left\{t^{\prime}=0\right\}$ and $N_{1}=\left\{t^{\prime}=\varphi_{2}(x)-\varphi_{1}(x)\right\}$. Assumption 4.12 implies that $\varphi:=\varphi_{2}-\varphi_{1}$ is a Morse-Bott function. Therefore, after changing the local coordinate system $x$ on $M$, we may assume 
that $\pi\left(\chi\left(p^{\prime}\right)\right)=(0,0)$ in the coordinates $\left(x, t^{\prime}\right)$ and $\varphi(x)=-x_{1}^{2}-\cdots-x_{\lambda}^{2}+x_{\lambda+1}^{2}+\cdots+x_{l}^{2}$, where $l:=\operatorname{dim} M-\operatorname{dim} C_{j}$. Note that in the coordinate system on $T^{*}(M \times \mathbb{R})$ associated with $\left(x, t^{\prime}\right)$, we have $\chi\left(p^{\prime}\right)=(0,0 ; 0,1)$. Hence by (2.19) we obtain

$$
\begin{aligned}
\mu_{N_{2}}\left(\mathbf{k}_{N_{1}}\right)_{\chi\left(p^{\prime}\right)} & \simeq \mu_{\mathbb{R}^{\operatorname{dim} M} \times\{0\}}\left(\mathbf{k}_{\left\{t^{\prime}=\varphi(x)\right\}}\right)_{(0,0 ; 0,1)} \\
& \simeq R \Gamma_{\left\{t^{\prime} \geq 0\right\}}\left(\mathbf{k}_{\left\{t^{\prime}=\varphi(x)\right\}}\right)_{0} \\
& \simeq \mathbf{k}[-\lambda] .
\end{aligned}
$$

Thus $\mu$ hom $\left.\left(F_{2}, F_{1}\right)\right|_{\widehat{C_{j}}}$ is concentrated in some degree and locally constant of rank 1 . Since $\mathbf{k}=\mathbb{F}_{2}$, a locally constant sheaf of rank 1 is constant, which implies the isomorphism $\left.\mu h o m\left(F_{2}, F_{1}\right)\right|_{\widehat{C_{j}}} \simeq \mathbf{k}_{\widehat{C_{j}}}\left[d_{1}(p)-d_{2}(p)-\delta_{1}+\delta_{2}-\lambda\right]$.

(b) We shall prove

$$
\lambda+\delta_{1}-\delta_{2}=\frac{1}{2}\left(\operatorname{dim} M-\operatorname{dim} C_{j}\right)-\frac{1}{2} \tau\left(\lambda_{L_{2}}(p), \lambda_{L_{1}}(p), \lambda_{\infty}(p)\right) .
$$

For the above coordinates $x$ on $M$, we set $x^{\prime}=\left(x_{1}, \ldots, x_{l}\right), x^{\prime \prime}=\left(x_{l+1}, \ldots, x_{m}\right)$ with $m=\operatorname{dim} M$ and denote by $(x ; \xi)=\left(x^{\prime}, x^{\prime \prime} ; \xi^{\prime}, \xi^{\prime \prime}\right)$ the associated coordinates on $T^{*} M$. We also denote by $\partial_{x, x}^{2} \varphi(0)=\left(\partial_{x_{j} x_{k}}^{2} \varphi(0)\right)_{j, k}$ the Hessian of $\varphi$. Then, by a similar argument to that of the proof of [KS90, Prop. 7.5.3], we get

$$
\begin{aligned}
\tau\left(\lambda_{\infty}(0), T_{0}\left(T_{\mathbb{R}^{m}}^{*} \mathbb{R}^{m}\right), T_{0} \Gamma_{d \varphi}\right) & =\tau\left(\{x=0\},\{\xi=0\},\left\{\xi=\partial_{x, x}^{2} \varphi(0) \cdot x\right\}\right) \\
& =\tau\left(\left\{x^{\prime}=0\right\},\left\{\xi^{\prime}=0\right\},\left\{\xi^{\prime}=\partial_{x^{\prime}, x^{\prime}}^{2} \varphi(0) \cdot x^{\prime}\right\}\right) \\
& =-\operatorname{sgn}\left(\partial_{x^{\prime}, x^{\prime}}^{2} \varphi(0)\right)=2 \lambda-l .
\end{aligned}
$$

Moreover, we have

$$
\begin{aligned}
& \tau\left(\chi\left(\lambda_{\Lambda_{2}}\left(p^{\prime}\right)\right), \chi\left(\lambda_{\Lambda_{1}}\left(p^{\prime}\right)\right), \lambda_{\infty}\left(\chi\left(p^{\prime}\right)\right)\right) \\
= & \tau\left(\lambda_{\widehat{T_{M}^{*} M}}\left(\chi\left(p^{\prime}\right)\right), \lambda_{\widehat{\Gamma_{-d \varphi}}}\left(\chi\left(p^{\prime}\right)\right), \lambda_{\infty}\left(\chi\left(p^{\prime}\right)\right)\right) \\
= & \tau\left(T_{0}\left(T_{\mathbb{R}^{m}}^{*} \mathbb{R}^{m}\right), T_{0} \Gamma_{-d \varphi}, \lambda_{\infty}(0)\right) \\
= & -\tau\left(\lambda_{\infty}(0), T_{0}\left(T_{\mathbb{R}^{m}}^{*} \mathbb{R}^{m}\right), T_{0} \Gamma_{d \varphi}\right) .
\end{aligned}
$$

Here we used the homogeneous symplectic coordinate system associated with $\left(x, t^{\prime}\right)$ for the first equality, Lemma C.2 for the second one, and Proposition C.1(i) for the last one. Combining the above two equalities, we finally obtain

$$
\begin{aligned}
-2 \lambda+l-2 \delta_{1}+2 \delta_{2}= & \tau\left(\chi\left(\lambda_{\Lambda_{2}}\left(p^{\prime}\right)\right), \chi\left(\lambda_{\Lambda_{1}}\left(p^{\prime}\right)\right), \lambda_{\infty}\left(\chi\left(p^{\prime}\right)\right)\right)-2 \delta_{1}+2 \delta_{2} \\
= & \tau\left(\lambda_{\Lambda_{2}}\left(p^{\prime}\right), \lambda_{\Lambda_{1}}\left(p^{\prime}\right), \chi^{-1}\left(\lambda_{\infty}\left(\chi\left(p^{\prime}\right)\right)\right)\right) \\
& +\tau\left(\lambda_{\Lambda_{1}}\left(p^{\prime}\right), \lambda_{\infty}\left(p^{\prime}\right), \chi^{-1}\left(\lambda_{\infty}\left(\chi\left(p^{\prime}\right)\right)\right)\right) \\
& +\tau\left(\lambda_{\infty}\left(p^{\prime}\right), \lambda_{\Lambda_{2}}\left(p^{\prime}\right), \chi^{-1}\left(\lambda_{\infty}\left(\chi\left(p^{\prime}\right)\right)\right)\right) \\
= & \tau\left(\lambda_{\Lambda_{2}}\left(p^{\prime}\right), \lambda_{\Lambda_{1}}\left(p^{\prime}\right), \lambda_{\infty}\left(p^{\prime}\right)\right) \\
= & \tau\left(\lambda_{L_{2}}(p), \lambda_{L_{1}}(p), \lambda_{\infty}(p)\right) .
\end{aligned}
$$

Here the second equality follows from the invariance under symplectic isomorphisms, the third one follows from the "cocycle condition" of the inertia index (Proposition C.1(ii)), and the last one follows from Lemma C.2 again. Since $l=\operatorname{dim} M-\operatorname{dim} C_{j}$, this completes the proof.

For a general filed $\mathbf{k}$, if $L_{1}$ and $L_{2}$ are the graphs of exact 1-forms and intersect cleanly, the locally constant object $\left.\mu h o m\left(F_{2}, F_{1}\right)\right|_{\Omega_{+}}$is described as follows: 
Proposition 4.15. Let $\mathbf{k}$ be any field. Under Assumption 4.12, assume, moreover, that there exists a $C^{\infty}$-function $\varphi_{i}: M \rightarrow \mathbb{R}$ such that $L_{i}=\Gamma_{d \varphi_{i}}$ and $f_{i}=\left.\varphi_{i} \circ \pi\right|_{L_{i}}$ for $i=1,2$. Define a Morse-Bott function $\varphi$ on $M$ by $\varphi:=\varphi_{2}-\varphi_{1}$ and let $C_{1}, \ldots, C_{n_{0}}$ be the critical components of $\varphi$ with $\varphi\left(C_{j}\right)=0\left(j=1, \ldots, n_{0}\right)$. For such a critical component $C_{j}$, define $T_{C_{j}}^{-} M$ as the maximal subbundle of $T_{C_{j}} M$ where the restriction of the Hessian $\left.\operatorname{Hess}(\varphi)\right|_{T_{C_{j}}^{-} M}$ is negative definite, and define a closed subset $\widehat{C_{j}}$ of $\Omega_{+}$by

$$
\widehat{C_{j}}:=\left\{\left(x,-\varphi_{1}(x) ; \tau d \varphi_{1}(x), \tau\right) \mid \tau>0, x \in C_{j}\right\} .
$$

Moreover, let $\mathcal{L}_{i}:=\left(F_{i}\right)_{+} \in \operatorname{Mod}\left(\mathbf{k}_{M}\right)$ be the locally constant sheaf of rank 1 associated with the simple sheaf quantization $F_{i}$ for $i=1,2$. Then

$$
\begin{aligned}
\left.\mu h o m\left(F_{2}, F_{1}\right)\right|_{\Omega_{+}} & \simeq \bigoplus_{j=1}^{n_{0}} \pi_{j}^{-1}\left(\omega_{C_{j} / T_{C_{j}}^{-} M} \otimes \mathcal{L}_{1} \otimes \mathcal{L}_{2}^{\otimes-1}\right) \\
& \simeq \bigoplus_{j=1}^{n_{0}} \pi_{j}^{-1}\left(\operatorname{or}_{C_{j} / T_{C_{j}}^{-} M} \otimes \mathcal{L}_{1} \otimes \mathcal{L}_{2}^{\otimes-1}\right)\left[-s\left(C_{j}\right)\right]
\end{aligned}
$$

where $\pi_{j}: \widehat{C_{j}} \rightarrow C_{j}$ is the projection, $s\left(C_{j}\right) \in \mathbb{Z}$ is the fiber dimension of $T_{C_{j}}^{-} M$, which is equal to $s\left(C_{j}\right)$ given by (4.46) in the statement of Theorem 4.14, and the right-hand sides denote their zero-extensions to $\Omega_{+}$by abuse of notation.

Proof. We may assume that $\varphi_{1} \equiv 0, \varphi_{2} \equiv \varphi$ and $\mathcal{L}_{i} \simeq \mathbf{k}_{M}$ for $i=1,2$. Then $F_{1} \simeq$ $\mathbf{k}_{M \times[0,+\infty)}$ and $F_{2} \simeq \mathbf{k}_{\{(x, t) \mid \varphi(x)+t \geq 0\}}$. Take a critical component $C_{j}$ of $\varphi$ satisfying $\varphi\left(C_{j}\right)=$ 0 . Then by Proposition 2.16 we have

$$
\begin{aligned}
& \left.\mu h o m\left(\mathbf{k}_{\{(x, t) \mid \varphi(x)+t \geq 0\}}, \mathbf{k}_{M \times[0,+\infty)}\right)\right|_{\widehat{C_{j}}} \\
\simeq & \left.\pi_{j}^{-1} R \Gamma_{\{(x, t) \mid \varphi(x)+t \geq 0\}}\left(\mathbf{k}_{M \times[0,+\infty)}\right)\right|_{C_{j} \times\{0\}} \\
\simeq & \left.\pi_{j}^{-1} R \Gamma_{\{(x, t) \mid \varphi(x)+t \geq 0\}}\left(\mathbf{k}_{M \times\{0\}}\right)\right|_{C_{j} \times\{0\}} \\
\simeq & \left.\pi_{j}^{-1} R \Gamma_{\{\varphi \geq 0\}}\left(\mathbf{k}_{M}\right)\right|_{C_{j}} .
\end{aligned}
$$

Moreover, we obtain (cf. [ST92, Cor. 1.3])

$$
\left.\left.R \Gamma_{\{\varphi \geq 0\}}\left(\mathbf{k}_{M}\right)\right|_{C_{j}} \simeq R \Gamma_{C_{j}}\left(\mathbf{k}_{T_{C_{j}}^{-} M}\right)\right|_{C_{j}} \simeq \omega_{C_{j} / T_{C_{j}}^{-} M},
$$

which completes the proof.

In the case $L_{1}$ and $L_{2}$ intersect transversally, we also obtain the following:

Proposition 4.16. Let $\mathbf{k}$ be any field and assume that $L_{1}$ and $L_{2}$ intersect transversally. For an intersection point $p \in L_{1} \cap L_{2}$ with $f_{2}(p)-f_{1}(p)=0$, define $\widehat{p}:=\left\{\left(\tau p,-f_{1}(p) ; \tau\right) \in\right.$ $\left.T^{*} M \times T^{*} \mathbb{R} \mid \tau>0\right\} \subset \Omega_{+}$as a special case of (4.44). Then

$$
\left.\operatorname{\mu hom}\left(F_{2}, F_{1}\right)\right|_{\Omega_{+}} \simeq \bigoplus_{\substack{p \in L_{1} \cap L_{2}, f_{2}(p)-f_{1}(p)=0}} \mathbf{k}_{\widehat{p}}[-s(p)],
$$

where $s(p) \in \mathbb{Z}$ is given by (4.46) in the statement of Theorem 4.14.

Proof. In this case, the support of $\mu$ hom $\left.\left(F_{2}, F_{1}\right)\right|_{\Omega_{+}}$is contained in $\bigsqcup_{p} \widehat{p}$ and each $\widehat{p}$ is contractible. Hence $\mu$ hom $\left.\left(F_{2}, F_{1}\right)\right|_{\Omega_{+}}$has constant cohomology sheaves on $\bigsqcup_{p} \widehat{p}$. The rest is exactly the same as the proof of Theorem 4.14 . 
The relation between the degree $s(C)$ and the Maslov index will be explored in Section $\mathrm{C}$,

Theorem 4.17. Under Assumption 4.12, let $L_{1} \cap L_{2}=\bigsqcup_{j=1}^{n} C_{j}$ be the decomposition into connected components. Recall that for a component $C$ of $L_{1} \cap L_{2}$, one defines $f_{21}(C):=$ $f_{2}(p)-f_{1}(p)$, taking some $p \in C$. Moreover, let $a, b \in \mathbb{R}$ with $a<b$ or $a \in \mathbb{R}, b=+\infty$. Then

$$
\sum_{a \leq f_{21}\left(C_{j}\right)<b} \operatorname{dim}_{\mathbb{F}_{2}} H^{k-s\left(C_{j}\right)}\left(C_{j} ; \mathbb{F}_{2}\right) \geq \operatorname{dim}_{\mathbb{F}_{2}} H^{k} R \Gamma_{M \times[a, b)}\left((-\infty, b) ; \mathcal{H o m}^{\star}\left(F_{2}, F_{1}\right)\right)
$$

for any $k \in \mathbb{Z}$, where $s\left(C_{j}\right)$ is given by (4.46) in the statement of Theorem 4.14. In particular,

$$
\sum_{j=1}^{n} \operatorname{dim}_{\mathbb{F}_{2}} H^{k-s\left(C_{j}\right)}\left(C_{j} ; \mathbb{F}_{2}\right) \geq \operatorname{dim}_{\mathbb{F}_{2}} \operatorname{Hom}_{\mathcal{T}(M)}\left(F_{2}, F_{1}[k]\right)
$$

for any $k \in \mathbb{Z}$. If $L_{1}$ and $L_{2}$ intersect transversally, the inequalities hold for any field $\mathbf{k}$, not only for $\mathbb{F}_{2}$.

Proof. Since the set $\left\{f_{2}(p)-f_{1}(p) \mid p \in L_{1} \cap L_{2}\right\} \subset \mathbb{R}$ is finite, conditions (1) and (2) in Proposition 4.11 are satisfied. Moreover, by Theorem 4.14, condition (3) is also satisfied. Hence the first assertion follows from Proposition 4.11 and Theorem 4.14. For the second assertion, by Proposition 3.9 it is enough to show that

$$
\sum_{j=1}^{n} \operatorname{dim}_{\mathbb{F}_{2}} H^{k-s\left(C_{j}\right)}\left(C_{j} ; \mathbb{F}_{2}\right) \geq \operatorname{dim}_{\mathbb{F}_{2}} \operatorname{Hom}_{\mathcal{D}(M)}\left(F_{2}, T_{c *} F_{1}[k]\right)
$$

for any $c \in \mathbb{R}$ and any $k \in \mathbb{Z}$. This follows from Proposition 3.4 and the first assertion for the case $a=0, b=+\infty$. The last assertion follows from Proposition 4.16,

Corollary 4.18 ([Nad09, Thm. 1.3.1] and [FSS08, Thm. 1]). Under Assumption 4.12 and in the same notation as in Theorem 4.17, one has

$$
\sum_{j=1}^{n} \sum_{k \in \mathbb{Z}} \operatorname{dim}_{\mathbb{F}_{2}} H^{k}\left(C_{j} ; \mathbb{F}_{2}\right) \geq \sum_{k \in \mathbb{Z}} \operatorname{dim}_{\mathbb{F}_{2}} H^{k}\left(M ; \mathbb{F}_{2}\right) \text {. }
$$

If $L_{1}$ and $L_{2}$ intersect transversally, then

$$
\#\left(L_{1} \cap L_{2}\right) \geq \sum_{k \in \mathbb{Z}} \operatorname{dim} H^{k}(M ; \mathcal{L})
$$

for any rank 1 locally constant sheaf $\mathcal{L} \in \operatorname{Mod}\left(\mathbf{k}_{M}\right)$ over any field $\mathbf{k}$.

Proof. It follows from Proposition 4.2 and Theorem 4.17 .

Remark 4.19. Assume $L_{1}=L_{2}=L$ and $f_{1}=f_{2}$, and set $\mathcal{L}_{i}:=\left(F_{i}\right)_{+}$for $i=1,2$. Then $\left\{\left.\mu h o m\left(T_{c *} F_{2}, F_{1}\right)\right|_{\Omega_{+}}\right\}_{c}$ is concentrated at $c=0$ and $\left.\mu h o m\left(F_{2}, F_{1}\right)\right|_{\Omega_{+}} \simeq \pi_{\widehat{L}}^{-1}\left(\mathcal{L}_{2} \otimes \mathcal{L}_{1}^{\otimes-1}\right)$, where $\pi_{\widehat{L}}: \widehat{L} \rightarrow M$ is the projection, over any field $\mathbf{k}$. Let $a, b \in \mathbb{R}$ with $a<b$ or $a \in \mathbb{R}, b=+\infty$. In this case, we obtain a more precise description of the complex 
$R \Gamma_{M \times[a, b)}\left(M \times(-\infty, b) ; \mathcal{H o m}{ }^{\star}\left(F_{2}, F_{1}\right)\right)$, not only the Morse-Bott-type inequality. Namely, if $a \leq 0<b$, using the concentration, Lemma 4.9, and Proposition 4.10, we have

$$
\begin{aligned}
& R \Gamma_{M \times[a, b)}\left(M \times(-\infty, b) ; \mathcal{H o m}^{\star}\left(F_{2}, F_{1}\right)\right) \\
\simeq & R \Gamma\left(M \times\{0\} ;\left.R \Gamma_{M \times[0,+\infty)}\left(\mathcal{H o m}^{\star}\left(F_{2}, F_{1}\right)\right)\right|_{M \times\{0\}}\right) \\
\simeq & R \Gamma\left(\Omega_{+} ;\left.\mu h o m\left(F_{2}, F_{1}\right)\right|_{\Omega_{+}}\right) \\
\simeq & R \Gamma\left(\widehat{L} ; \pi_{\widehat{L}}^{-1}\left(\mathcal{L}_{2} \otimes \mathcal{L}_{1}^{\otimes-1}\right)\right) .
\end{aligned}
$$

This is essentially one of the results of Guillermou [Gui12, Thm. 20.4].

\section{A Degenerate Lagrangian intersections}

In this section, using very simple examples, we briefly remark that our method can also deal with degenerate Lagrangian intersections. Until the end of this section we set $\mathbf{k}=\mathbb{Q}$. We shall consider $T^{*} S^{1}$ and the intersection of the zero-section $S^{1}$ and the graph of an exact 1-form $L=\Gamma_{d f}$. Let $F:=\mathbf{k}_{S^{1} \times[0,+\infty)}$ be the canonical sheaf quantization associated with the zero-section $S^{1}$ and $G:=\mathbf{k}_{\left\{(x, t) \in S^{1} \times \mathbb{R} \mid f(x)+t \geq 0\right\}}$ be that associated with $L$. Assume that the intersection of $S^{1}$ and $L$ has only one possibly degenerate component $C$ and it is transversal outside $C$. Then by Proposition 4.11 and a similar argument to the proof of Theorem 4.17 we obtain

$$
\begin{aligned}
& \#\left\{p \in S^{1} \cap L \mid p \text { is a transverse intersection point }\right\} \\
& +\sum_{k \in \mathbb{Z}} \operatorname{dim} H^{k} R \Gamma\left(\Omega_{+} \cap \pi^{-1}(C) ;\left.\mu h o m(F, G)\right|_{\Omega_{+} \cap \pi^{-1}(C)}\right) \\
& \quad \geq \sum_{k} \operatorname{dim} \operatorname{Hom}_{\mathcal{T}\left(S^{1}\right)}(F, G[k])=\sum_{k} \operatorname{dim} H^{k}\left(S^{1} ; \mathbf{k}_{S^{1}}\right)=2 .
\end{aligned}
$$

We calculate the "contribution" $R \Gamma\left(\Omega_{+} \cap \pi^{-1}(C) ;\left.\mu h o m(F, G)\right|_{\Omega_{+} \cap \pi^{-1}(C)}\right)$ from $C$ in the following two typical examples.

First we consider the case that the intersection is as in Figure A.1 in a neighborhood of $C$. In this case, $G$ is isomorphic to the constant sheaf supported in the shaded closed subset in Figure A.2 in a neighborhood of $C$.

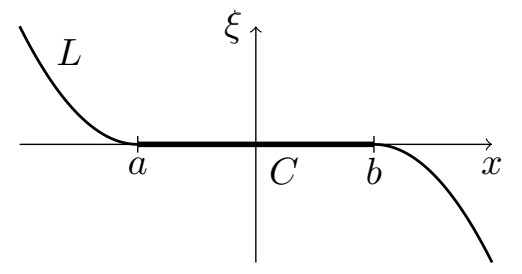

Figure A.1: $L$ in the first example

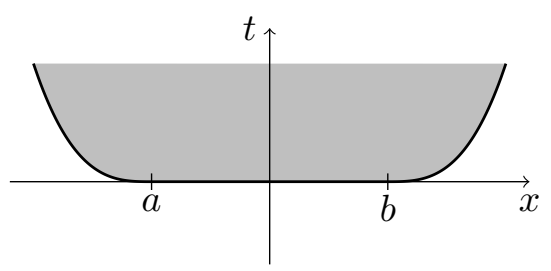

Figure A.2: $G$ in the first example

Hence we find that $\left.\mu h o m(F, G)\right|_{\Omega_{+} \cap \pi^{-1}(C)} \simeq \mathbf{k}_{[a, b] \times(0,+\infty)}$ and

$$
R \Gamma\left(\Omega_{+} \cap \pi^{-1}(C) ;\left.\mu h o m(F, G)\right|_{\Omega_{+} \cap \pi^{-1}(C)}\right) \simeq R \Gamma\left([a, b] ; \mathbf{k}_{[a, b]}\right) \simeq \mathbf{k} .
$$

Thus in this case the contribution from $C$ is 1 in (A.1), and the cardinality of the transverse intersection points is at least 1 , as expected.

Next we consider the case that the intersection is as in Figure A.3 in a neighborhood of $C$. The canonical sheaf quantization $G$ associated with $L$ is isomorphic to the constant sheaf supported in the shaded closed subset in Figure A.4 in a neighborhood of $C$. 


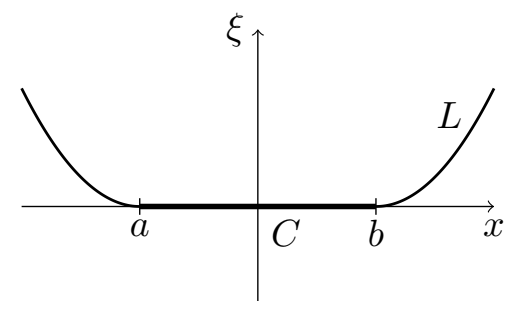

Figure A.3: $L$ in the second example

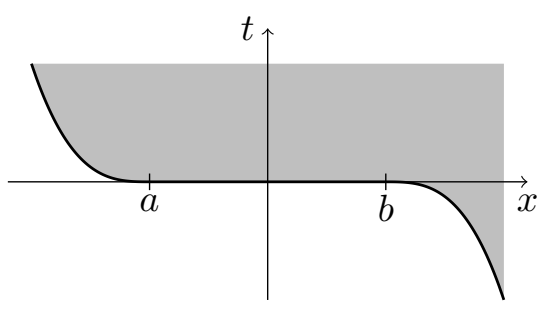

Figure A.4: $G$ in the second example

Therefore, in this case we get $\left.\mu h o m(F, G)\right|_{\Omega_{+} \cap \pi^{-1}(C)} \simeq \mathbf{k}_{[a, b) \times(0,+\infty)}$ and

$$
R \Gamma\left(\Omega_{+} \cap \pi^{-1}(C) ;\left.\mu h o m(F, G)\right|_{\Omega_{+} \cap \pi^{-1}(C)}\right) \simeq R \Gamma_{c}\left([a, b) ; \mathbf{k}_{[a, b)}\right) \simeq 0 .
$$

Hence the contribution from $C$ is 0 in (A.1) and the cardinality of the transverse intersection points is at least 2 in the second case.

Remark A.1. For $i=1,2$, let $L_{i}$ be a compact connected exact Lagrangian submanifold and $f_{i}: L_{i} \rightarrow \mathbb{R}$ be a function satisfying $d f_{i}=\left.\alpha\right|_{L_{i}}$. Moreover, let $F_{i}$ be a simple sheaf quantization associated with $L_{i}$ and $f_{i}$. Proposition 4.11 says that the contribution from components on which $f_{2}(p)-f_{1}(p)=c$ is encoded in the sheaf $\left.\mu h o m\left(T_{c *} F_{2}, F_{1}\right)\right|_{\Omega_{+}}$(even for possibly degenerate Lagrangian intersections). If the intersection is clean along a component $C$, then $\mu$ hom $\left.\left(T_{c *} F_{2}, F_{1}\right)\right|_{\Omega_{+}}$is locally constant of rank 1 on the cone of $C$ as in Lemma 4.13. However, as seen in the above examples, if the intersection is degenerate, then $\left.\operatorname{\mu hom}\left(T_{c *} F_{2}, F_{1}\right)\right|_{\Omega_{+}}$is not necessarily locally constant.

\section{B Functoriality of sheaf quantizations}

In this section we prove the "functoriality" of Guillermou's simple sheaf quantizations with respect to Hamiltonian diffeomorphisms. We remark that results in this section are independent of the results in Section 4 and not used for the proofs of them.

Let $L$ be a compact connected exact Lagrangian submanifold of $T^{*} M$ and $f$ be a primitive of the Liouville form $\alpha$. We define the conification $\widehat{L}_{f}$ of $L$ with respect to $f$ as in (3.20). Let $\psi$ be a Hamiltonian diffeomorphism of $T^{*} M$ and $\phi=\left(\phi_{s}\right)_{s}: T^{*} M \times I \rightarrow T^{*} M$ be a Hamiltonian isotopy, where $I$ is an open interval containing $[0,1]$, such that $\phi_{1}=\psi$ and $\phi_{0}=\mathrm{id}_{T^{*} M}$. We denote by $H=\left(H_{s}\right)_{s}: T^{*} M \times I \rightarrow \mathbb{R}$ the associated Hamiltonian and by $X_{s}$ the associated Hamiltonian vector field on $T^{*} M$. The homogeneous lift $\widehat{\phi}$ of $\phi$ is described as follows (see [GKS12, Prop. A.6]):

$$
\widehat{\phi}_{1}(x, t ; \xi, \tau)=\left(x^{\prime}, t+u(x ; \xi / \tau) ; \xi^{\prime}, \tau\right),
$$

where $\left(x^{\prime} ; \xi^{\prime} / \tau\right)=\phi_{1}(x ; \xi / \tau)=\psi(x ; \xi / \tau)$ and $u: T^{*} M \rightarrow \mathbb{R}$ is defined by

$$
u(p)=\int_{0}^{1}\left(H_{s}-\alpha\left(X_{s}\right)\right)\left(\phi_{s}(p)\right) d s .
$$

Hence we get

$$
\begin{aligned}
\widehat{\phi}_{1}\left(\widehat{L}_{f}\right) & =\left\{\begin{array}{l|l}
\left(x^{\prime}, t+u(x ; \xi / \tau) ; \xi^{\prime}, \tau\right) & \begin{array}{l}
\tau>0, \exists(x ; \xi) \text { s.t. }(x ; \xi / \tau) \in L, \\
\left(x^{\prime} ; \xi^{\prime} / \tau\right)=\psi(x ; \xi / \tau), t=-f(x ; \xi / \tau)
\end{array}
\end{array}\right\} \\
& =\left\{\begin{array}{l|l}
\left(x^{\prime}, t^{\prime} ; \xi^{\prime}, \tau\right) & \begin{array}{l}
\tau>0,\left(x^{\prime} ; \xi^{\prime} / \tau\right) \in \psi(L), \\
t^{\prime}=-f \circ \psi^{-1}\left(x^{\prime} ; \xi^{\prime} / \tau\right)+u \circ \psi^{-1}\left(x^{\prime} ; \xi^{\prime} / \tau\right)
\end{array}
\end{array}\right\} .
\end{aligned}
$$


On the other hand, we have equalities

$$
\begin{aligned}
\psi^{*} \alpha-\alpha & =\int_{0}^{1}\left(\frac{d}{d s} \phi_{s}^{*} \alpha\right) d s \\
& =\int_{0}^{1} \phi_{s}^{*}\left(L_{X_{s}} \alpha\right) d s \\
& =\int_{0}^{1} \phi_{s}^{*}\left(d \iota_{X_{s}} \alpha+\iota_{X_{s}} d \alpha\right) d s \\
& =d \int_{0}^{1} \phi_{s}^{*}\left(\alpha\left(X_{s}\right)-H_{s}\right) d s=-d u .
\end{aligned}
$$

Here, for a vector field $X, L_{X}$ denotes the Lie derivative with respect to $X$, and the third equality follows from Cartan's formula. Moreover, the fourth equality follows from the definition of the Hamiltonian vector field: $d \alpha\left(X_{s}, *\right)=-d H_{s}$. Hence setting $\widetilde{f}:=$ $(f-u) \circ \psi^{-1}: \psi(L) \rightarrow \mathbb{R}$, we get

$$
\begin{aligned}
\left.\alpha\right|_{\psi(L)} & =\left(\psi^{-1}\right)^{*}\left(\left.\alpha\right|_{L}-\left.d u\right|_{L}\right) \\
& =\left(\psi^{-1}\right)^{*}\left(d f-\left.d u\right|_{L}\right)=d \widetilde{f} .
\end{aligned}
$$

Thus we find that $\tilde{f}$ is a primitive of $\alpha$ on $\psi(L)$ and obtain the following:

Lemma B.1. One has

$$
\widehat{\phi}_{1}\left(\widehat{L}_{f}\right)=\widehat{\psi(L)}_{\widetilde{f}} \subset T^{*}(M \times \mathbb{R}) .
$$

Proposition B.2. Let $\mathcal{L} \in \operatorname{Mod}\left(\mathbf{k}_{M}\right)$ be a locally constant sheaf of rank 1 and $F_{L}$ be the simple sheaf quantization of $\widehat{L}_{f}$ satisfying $F_{L_{+}} \simeq \mathcal{L}$. Let $\psi: T^{*} M \rightarrow T^{*} M$ be a Hamiltonian diffeomorphism and $\Psi: \mathbf{D}^{\mathrm{b}}(M \times \mathbb{R}) \rightarrow \mathbf{D}^{\mathrm{b}}(M \times \mathbb{R})$ the associated functor (see (3.15) ). Define $\tilde{f}:=(f-u) \circ \psi^{-1}: \psi(L) \rightarrow \mathbb{R}$ as above and denote by $\widehat{\psi(L)}_{\tilde{f}}$ the conification of $\psi(L)$ with respect to $\tilde{f}$. Moreover, let $F_{\psi(L)}$ be the simple sheaf quantization of $\widehat{\psi(L)}_{\widetilde{f}}$ satisfying $F_{\psi(L)_{+}} \simeq \mathcal{L}$. Then

$$
\Psi\left(F_{L}\right) \simeq F_{\psi(L)}
$$

Proof. By Lemma B.1, we have

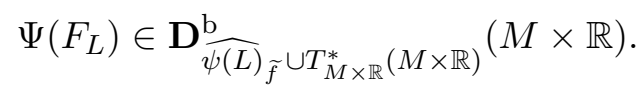

By the uniqueness of simple sheaf quantizations (Theorem 3.13), it remains to show that

$$
\Psi\left(F_{L}\right)_{-} \simeq 0, \quad \Psi\left(F_{L}\right)_{+} \simeq \mathcal{L}
$$

Let $\widehat{\phi}: \stackrel{\circ}{T}^{*}(M \times \mathbb{R}) \times I \rightarrow \stackrel{\circ}{T}^{*}(M \times \mathbb{R})$ be the associated homogeneous Hamiltonian isotopy and $K \in \mathbf{D}^{\mathrm{lb}}(M \times \mathbb{R} \times M \times \mathbb{R} \times I)$ be the sheaf quantization of $\widehat{\phi}$. Let $\varepsilon \in \mathbb{R}_{>0}$ satisfying $[-\varepsilon, 1+\varepsilon] \subset I$. By the compactness of $L$, there exists $A \in \mathbb{R}_{>0}$ satisfying

$$
\bigcup_{s \in[-\varepsilon, 1+\varepsilon]} \widehat{\phi}_{s}\left(\widehat{L}_{f}\right) \subset T^{*}(M \times(-A, A)) .
$$


Replacing $I$ with the relatively compact subinterval $(-\varepsilon, 1+\varepsilon)$, we may assume that

$$
\bigcup_{s \in I} \widehat{\phi}_{s}\left(\widehat{L}_{f}\right) \subset T^{*}(M \times(-A, A))
$$

and $K \in \mathbf{D}^{\mathrm{b}}(M \times \mathbb{R} \times M \times \mathbb{R} \times I)$ from the beginning. Set $G:=\left.\left(K \circ F_{L}\right)\right|_{M \times(A,+\infty) \times I} \in$ $\mathbf{D}^{\mathrm{b}}(M \times(A,+\infty) \times I)$. We shall show that

$$
\mathrm{SS}(G) \subset T_{M \times(A,+\infty) \times I}^{*}(M \times(A,+\infty) \times I) .
$$

First, by Proposition 2.11, we have

$$
\operatorname{SS}\left(K \circ F_{L}\right) \subset\left(\Lambda_{\widehat{\phi}} \circ \widehat{L}_{f}\right) \cup T_{M \times \mathbb{R} \times I}^{*}(M \times \mathbb{R} \times I) .
$$

By the definition of $\Lambda_{\widehat{\phi}}($ see $(\underline{3.3}))$, we obtain

$$
\left(\Lambda_{\widehat{\phi}} \circ \widehat{L}\right) \cap\left(T_{M \times \mathbb{R}}^{*}(M \times \mathbb{R}) \times T^{*} I\right) \subset T_{M \times \mathbb{R} \times I}^{*}(M \times \mathbb{R} \times I) .
$$

Denote by $i_{s}: M \times \mathbb{R} \times\{s\} \hookrightarrow M \times \mathbb{R} \times I$ the closed embedding for any $s \in I$. Then, by the definition of $\Lambda_{\widehat{\phi}}$, we also have

$$
\left(i_{s}\right)_{d}\left(i_{s}\right)_{\pi}^{-1}\left(\Lambda_{\widehat{\phi}} \circ \widehat{L}_{f}\right)=\widehat{\phi}_{s}\left(\widehat{L}_{f}\right) .
$$

Moreover by (B.9), we get

$$
\widehat{\phi}_{s}\left(\widehat{L}_{f}\right) \cap T^{*}(M \times(A,+\infty))=\emptyset
$$

for any $s \in I$. Hence the inclusion (B.10) follows from the above estimates (B.12), (B.13), and (B.14). Since $I$ is contractible, we have $G \simeq q^{-1}\left(\left.G\right|_{M \times(A,+\infty) \times\{0\}}\right)$, where $q: M \times$ $(A,+\infty) \times I \rightarrow M \times(A,+\infty)$ is the projection. In particular, we get

$$
\begin{aligned}
\left.\Psi\left(F_{L}\right)\right|_{M \times(A,+\infty)} & =\left.G\right|_{M \times(A,+\infty) \times\{1\}} \\
& \left.\simeq G\right|_{M \times(A,+\infty) \times\{0\}} \\
& \left.\simeq\left(F_{L}\right)\right|_{M \times(A,+\infty)} \simeq \mathcal{L} \otimes \mathbf{k}_{(A,+\infty)}
\end{aligned}
$$

and $\Psi\left(F_{L}\right)_{+} \simeq \mathcal{L}$. A similar argument shows that $\Psi\left(F_{L}\right)_{-} \simeq 0$.

\section{Relation to grading in Lagrangian Floer cohomology the- ory, by Tomohiro Asano}

In this section we relate the absolute grading of $\mathcal{H o m}^{\star}$ to that of Lagrangian Floer cohomology.

\section{C.1 Inertia index and Maslov index}

In this subsection we recall some properties of the inertia index and the Maslov index. First we list some properties of the inertia index.

Proposition C.1 ([KS90, Thm. A.3.2]). Let $E$ be a symplectic vector space and denote by $\mathcal{L}(E)$ the Lagrangian Grassmannian of $E$. The inertia index $\tau: \mathcal{L}(E)^{3} \rightarrow \mathbb{Z}$ satisfies the following properties. 
(i) For any $\lambda_{1}, \lambda_{2}, \lambda_{3} \in \mathcal{L}(E), \tau\left(\lambda_{1}, \lambda_{2}, \lambda_{3}\right)=-\tau\left(\lambda_{2}, \lambda_{1}, \lambda_{3}\right)=-\tau\left(\lambda_{1}, \lambda_{3}, \lambda_{2}\right)$.

(ii) The inertia index satisfies the "cocycle condition": for any quadruple $\lambda_{1}, \lambda_{2}, \lambda_{3}, \lambda_{4} \in$ $\mathcal{L}(E)$,

$$
\tau\left(\lambda_{1}, \lambda_{2}, \lambda_{3}\right)=\tau\left(\lambda_{1}, \lambda_{2}, \lambda_{4}\right)+\tau\left(\lambda_{2}, \lambda_{3}, \lambda_{4}\right)+\tau\left(\lambda_{3}, \lambda_{1}, \lambda_{4}\right)
$$

(iii) If $\lambda_{1}, \lambda_{2}, \lambda_{3}$ move continuously in the Lagrangian Grassmannian $\mathcal{L}(E)$ so that $\operatorname{dim}\left(\lambda_{1} \cap \lambda_{2}\right), \operatorname{dim}\left(\lambda_{2} \cap \lambda_{3}\right), \operatorname{dim}\left(\lambda_{3} \cap \lambda_{1}\right)$ remain constant, then $\tau\left(\lambda_{1}, \lambda_{2}, \lambda_{3}\right)$ remains constant.

(iv) Let $E^{\prime}$ be another symplectic vector space, and let $\lambda_{1}, \lambda_{2}, \lambda_{3}$ (resp. $\left.\lambda_{1}^{\prime}, \lambda_{2}^{\prime}, \lambda_{3}^{\prime}\right)$ be a triple of Lagrangian subspaces of $E$ (resp. $\left.E^{\prime}\right)$. Then

$$
\tau_{E \oplus E^{\prime}}\left(\lambda_{1} \oplus \lambda_{1}^{\prime}, \lambda_{2} \oplus \lambda_{2}^{\prime}, \lambda_{3} \oplus \lambda_{3}^{\prime}\right)=\tau_{E}\left(\lambda_{1}, \lambda_{2}, \lambda_{3}\right)+\tau_{E^{\prime}}\left(\lambda_{1}^{\prime}, \lambda_{2}^{\prime}, \lambda_{3}^{\prime}\right) .
$$

Let $M$ be a compact connected manifold without boundary and $T^{*} M$ be its cotangent bundle. Moreover, let $\mathcal{L}_{T^{*} M}$ be the fiber bundle over $T^{*} M$ whose fiber is the Lagrangian Grassmannian, that is, $\mathcal{L}_{T^{*} M, p}=\mathcal{L}\left(T_{p} T^{*} M\right)$. Denote by $\lambda_{\infty}: T^{*} M \rightarrow \mathcal{L}_{T^{*} M}, p \mapsto$ $T_{p} T_{\pi(p)}^{*} M$ be the section which assigns the fiber to $p$. A Lagrangian submanifold $L$ of $T^{*} M$ defines a section $\lambda_{L}: L \rightarrow \mathcal{L}_{T^{*} M}, p \mapsto T_{p} L$ over $L$.

Lemma C.2. For $i=1,2$, let $L_{i}$ be a compact connected exact Lagrangian submanifold and $f_{i}: L_{i} \rightarrow \mathbb{R}$ be a function such that $d f_{i}=\left.\alpha\right|_{L_{i}}$ and set $\Lambda_{i}:=\widehat{L}_{i f_{i}}$, the conification of $L_{i}$ with respect to $f_{i}$. Let $p \in L_{1} \cap L_{2}$ and assume $f_{1}(p)=f_{2}(p)$. Set $p^{\prime}:=\left(p,-f_{1}(p) ; 1\right) \in$ $\Lambda_{1} \cap \Lambda_{2} \subset T^{*}(M \times \mathbb{R})$. Then

$$
\tau_{T_{p^{\prime}} T^{*}(M \times \mathbb{R})}\left(\lambda_{\Lambda_{2}}\left(p^{\prime}\right), \lambda_{\Lambda_{1}}\left(p^{\prime}\right), \lambda_{\infty}\left(p^{\prime}\right)\right)=\tau_{T_{p} T^{*} M}\left(\lambda_{L_{2}}(p), \lambda_{L_{1}}(p), \lambda_{\infty}(p)\right) .
$$

Proof. Take a local homogeneous symplectic coordinate system $(x, t ; \xi, \tau)$ on $T^{*}(M \times \mathbb{R})$. Using the coordinate system, we identify $T_{p^{\prime}} T^{*}(M \times \mathbb{R})$ with $\mathbb{R}^{m} \times \mathbb{R} \times \mathbb{R}^{m} \times \mathbb{R}$. In this coordinate system, we get $\lambda_{\infty}\left(p^{\prime}\right)=0 \times 0 \times \mathbb{R}^{m} \times \mathbb{R}$. Write $p=(x ; \xi)$ using the coordinate system. Then $\lambda_{\Lambda_{i}}\left(p^{\prime}\right)$ is spanned by

$$
(0,0 ; \xi, 1),\left(v_{i},-T f_{i}\left(v_{i}\right) ; \zeta_{i}, 0\right) \quad\left(\left(v_{i}, \zeta_{i}\right) \in T_{p} L_{i}\right) .
$$

For $r \in[0,1]$, let $\lambda_{\Lambda_{i}}\left(p^{\prime} ; r\right)$ be the Lagrangian linear subspace spanned by

$$
(0,0 ; r \xi, 1),\left(v_{i},-r \cdot T f_{i}\left(v_{i}\right) ; \zeta_{i}, 0\right) \quad\left(\left(v_{i}, \zeta_{i}\right) \in T_{p} L_{i}\right) .
$$

Then by Proposition C.1(iii) we have

$$
\tau_{T_{p^{\prime}} T^{*}(M \times \mathbb{R})}\left(\lambda_{\Lambda_{2}}\left(p^{\prime}\right), \lambda_{\Lambda_{1}}\left(p^{\prime}\right), \lambda_{\infty}\left(p^{\prime}\right)\right)=\tau_{T_{p^{\prime}} T^{*}(M \times \mathbb{R})}\left(\lambda_{\Lambda_{2}}\left(p^{\prime} ; r\right), \lambda_{\Lambda_{1}}\left(p^{\prime} ; r\right), \lambda_{\infty}\left(p^{\prime}\right)\right)
$$

for any $r \in[0,1]$. Since $\lambda_{\Lambda_{i}}\left(p^{\prime} ; 0\right)=\lambda_{L_{i}}(p) \oplus \mathbb{R}\langle(0 ; 1)\rangle$, by Proposition C.1(iv), we obtain

$$
\begin{aligned}
& \tau_{T_{p^{\prime}} T^{*}(M \times \mathbb{R})}\left(\lambda_{\Lambda_{2}}\left(p^{\prime}\right), \lambda_{\Lambda_{1}}\left(p^{\prime}\right), \lambda_{\infty}\left(p^{\prime}\right)\right) \\
= & \tau_{T_{p^{\prime}} T^{*}(M \times \mathbb{R})}\left(\lambda_{\Lambda_{2}}\left(p^{\prime} ; 0\right), \lambda_{\Lambda_{1}}\left(p^{\prime} ; 0\right), \lambda_{\infty}\left(p^{\prime}\right)\right) \\
= & \tau_{T_{p} T^{*} M}\left(\lambda_{L_{2}}(p), \lambda_{L_{1}}(p), \lambda_{\infty}(p)\right) .
\end{aligned}
$$


Next we recall some properties of the Maslov index (see, for example, Leray [Ler81, Robbin-Salamon [RS93, and de Gosson dG09]).

Proposition C.3. Let $E$ be a symplectic vector space and denote by $\widetilde{\mathcal{L}}(E)$ the universal covering of the Lagrangian Grassmannian $\mathcal{L}(E)$ of $E$. For $\widetilde{\lambda_{i}} \in \widetilde{\mathcal{L}}(E)(i \in \mathbb{N})$, denote its projection to $\mathcal{L}(E)$ by $\lambda_{i}$. The Maslov index $\mu: \widetilde{\mathcal{L}}(E)^{2} \rightarrow \frac{1}{2} \mathbb{Z}$ satisfies the following properties.

(i) For any $\widetilde{\lambda_{1}}, \widetilde{\lambda_{2}} \in \widetilde{\mathcal{L}}(E), \mu\left(\widetilde{\lambda_{1}}, \widetilde{\lambda_{2}}\right)=-\mu\left(\widetilde{\lambda_{2}}, \widetilde{\lambda_{1}}\right)$

(ii) The coboundary of $\mu$ is given by $\tau: \mu\left(\widetilde{\lambda_{1}}, \widetilde{\lambda_{2}}\right)+\mu\left(\widetilde{\lambda_{2}}, \widetilde{\lambda_{3}}\right)+\mu\left(\widetilde{\lambda_{3}}, \widetilde{\lambda_{1}}\right)=\frac{1}{2} \tau\left(\lambda_{1}, \lambda_{2}, \lambda_{3}\right)$

(iii) If $\widetilde{\lambda_{1}}$ and $\widetilde{\lambda_{2}}$ move continuously in $\widetilde{\mathcal{L}}(E)$ so that $\operatorname{dim}\left(\lambda_{1} \cap \lambda_{2}\right)$ remains constant, then $\mu\left(\widetilde{\lambda_{1}}, \widetilde{\lambda_{2}}\right)$ remains constant.

(iv) For any $\widetilde{\lambda_{1}}, \widetilde{\lambda_{2}} \in \widetilde{\mathcal{L}}(E), \mu\left(\widetilde{\lambda_{1}}, \widetilde{\lambda_{2}}\right) \equiv \frac{1}{2}\left(\operatorname{dim}\left(\lambda_{1} \cap \lambda_{2}\right)+\frac{1}{2} \operatorname{dim} E\right) \bmod \mathbb{Z}$.

(v) Under an isomorphism $\rho: \pi_{1}(\mathcal{L}(E)) \simeq \mathbb{Z}$, for any $\widetilde{\lambda_{1}}, \widetilde{\lambda_{2}} \in \widetilde{\mathcal{L}}(E)$ and $n, m \in \mathbb{Z}$, $\mu\left(\rho^{-1}(n) \cdot \widetilde{\lambda_{1}}, \rho^{-1}(m) \cdot \widetilde{\lambda_{2}}\right)=\mu\left(\widetilde{\lambda_{1}}, \widetilde{\lambda_{2}}\right)+n-m$, where dots stand for the covering transformation.

Remark C.4. Notation for the Maslov index differs between authors. Our $\mu$ is equal to half of the $\mu$ in dG09. Note that (ii) and (iii) of the above proposition determine the function $\mu: \widetilde{\mathcal{L}}(E)^{2} \rightarrow \frac{1}{2} \mathbb{Z}$ uniquely.

\section{C.2 Graded Lagrangian submanifolds and Maslov index}

Next we recall the notion of graded Lagrangian submanifolds due to Seidel Sei00]. Denote by $\widetilde{\mathcal{L}}_{T^{*} M}$ the fiberwise universal cover of $\mathcal{L}_{T^{*} M}$ whose fiber over $p$ is identified with the space of the homotopy classes of paths in $\mathcal{L}_{T^{*} M, p}$ from $\lambda_{\infty}$. We also denote by $\mu: \widetilde{\mathcal{L}}_{T^{*} M} \times_{T^{*} M} \widetilde{\mathcal{L}}_{T^{*} M} \rightarrow \frac{1}{2} \mathbb{Z}$ the Maslov index on $T^{*} M$. For a Lagrangian submanifold $L$ of $T^{*} M$, a grading of $L$ is a lift $\widetilde{\lambda}: L \rightarrow \widetilde{\mathcal{L}}_{T^{*} M}$ of $\lambda_{L}$. A graded Lagrangian submanifold is a pair $(L, \widetilde{\lambda})$ consisting of a Lagrangian submanifold $L$ and a grading $\widetilde{\lambda}$ of $L$.

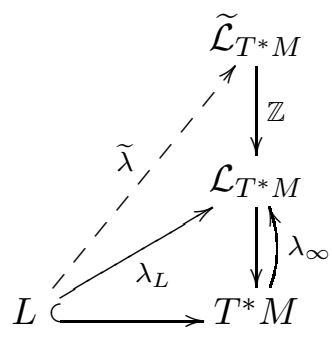

Now, let $\left(L_{1}, \widetilde{\lambda_{1}}\right)$ and $\left(L_{2}, \widetilde{\lambda_{2}}\right)$ be graded Lagrangian submanifolds of $T^{*} M$ intersecting cleanly. For a connected component $C$ of $L_{1} \cap L_{2}$, we define the absolute grading $\operatorname{gr}\left(L_{2}, L_{1} ; C\right)$ of $C$ by taking $p \in C$ and

$$
\operatorname{gr}\left(L_{2}, L_{1} ; C\right)=\frac{1}{2}(\operatorname{dim} M-\operatorname{dim} C)-\mu\left(\widetilde{\lambda_{2}}(p), \widetilde{\lambda_{1}}(p)\right),
$$


which induces the absolute grading of Lagrangian Floer cohomology. Note that by Proposition C.3 (i) and (ii), the grading $\operatorname{gr}\left(L_{2}, L_{1} ; C\right)$ is written as

$$
\begin{aligned}
& \operatorname{gr}\left(L_{2}, L_{1} ; C\right) \\
& =\frac{1}{2}(\operatorname{dim} M-\operatorname{dim} C)+\mu\left(\widetilde{\lambda_{1}}(p), \lambda_{\infty}(p)\right)+\mu\left(\lambda_{\infty}(p), \widetilde{\lambda_{2}}(p)\right)-\frac{1}{2} \tau\left(\lambda_{2}(p), \lambda_{1}(p), \lambda_{\infty}(p)\right) \\
& =\frac{1}{2}(\operatorname{dim} M-\operatorname{dim} C)+\mu\left(\lambda_{\infty}(p), \widetilde{\lambda_{2}}(p)\right)-\mu\left(\lambda_{\infty}(p), \widetilde{\lambda_{1}}(p)\right)-\frac{1}{2} \tau\left(T_{p} L_{2}, T_{p} L_{1}, \lambda_{\infty}(p)\right),
\end{aligned}
$$

where the point $\lambda_{\infty}(p)$ is regarded as (the homotopy class of) the constant path.

\section{C.3 Shifts of simple sheaf quantizations}

Let $L$ be a compact exact Lagrangian submanifold of $T^{*} M$ and $f: L \rightarrow \mathbb{R}$ be a primitive of the Liouville 1-form. Denote by $\widehat{L} \subset T^{*}(M \times \mathbb{R})$ the conification of $L$ with respect to $f$ and let $F \in \mathbf{D}^{\mathrm{b}}(M \times \mathbb{R})$ be a simple sheaf quantization of $\widehat{L}$. By Theorem 3.13, the object $F$ is simple along $\widehat{L}$ and the shift of $F$ at a point of $\widehat{L}$ defines a function $d: \widehat{L} \rightarrow \frac{1}{2} \mathbb{Z}$. Since $d\left(c \cdot p^{\prime}\right)=d\left(p^{\prime}\right)$ for any $p^{\prime} \in \widehat{L}$ and $c \in \mathbb{R}_{>0}$, and $\widehat{L} / \mathbb{R}_{>0}=L$, we also regard $d$ as a function $L \rightarrow \frac{1}{2} \mathbb{Z}$.

Proposition C.5. There is a grading $\tilde{\lambda}: L \rightarrow \widetilde{\mathcal{L}}_{T^{*} M}$ such that

$$
\mu\left(\lambda_{\infty}(p), \widetilde{\lambda}(p)\right)+\frac{1}{2}(\operatorname{dim} M+1)=d(p)
$$

where $\lambda_{\infty}$ denotes the constant path.

Proof. Let $\left.U_{L} \subset \mathcal{L}_{T^{*} M}\right|_{L}$ be the open subset of the Lagrangian Grassmannian restricted over $L$ consisting of Lagrangian subspaces transversal to $\lambda_{\infty}$ and $\lambda_{L}$. Moreover, let $U \subset$ $U_{L}$ be a connected open subset of $U_{L}$ whose image $\pi(U)$ under $\pi$ is contractible. Note that the set of such $\pi(U)$ covers $L$. For $p \in L$, we set $p^{\prime}:=(p,-f(p) ; 1) \in \widehat{L}$. Fix a local section $\gamma: \pi(U) \rightarrow U$ and take a local section $\gamma^{\prime}:\left.\rho^{-1}(\pi(U)) \rightarrow \mathcal{L}_{T^{*}(M \times \mathbb{R})}\right|_{\widehat{L}}$ so that $\gamma^{\prime}\left(p^{\prime}\right)=\gamma(p) \oplus \mathbb{R}\langle(1 ; 0)\rangle \subset T_{p} T^{*} M \oplus T_{(-f(p) ; 1)} T^{*} \mathbb{R}$ holds for every $p \in \pi(U)$. By Proposition C.3 and the same homotopy $\lambda_{\widehat{L}}\left(p^{\prime} ; r\right)$ as in the proof of Lemma C.2, we get

$$
\frac{1}{2} \tau\left(\lambda_{\infty}\left(p^{\prime}\right), \lambda_{\widehat{L}}\left(p^{\prime}\right), \gamma^{\prime}\left(p^{\prime}\right)\right)=\mu\left(\lambda_{\infty}(p), \widetilde{\lambda}(p)\right)+\mu(\widetilde{\lambda}(p), \widetilde{\gamma}(p))+\mu\left(\widetilde{\gamma}(p), \lambda_{\infty}(p)\right),
$$

where $\widetilde{\gamma}$ and $\tilde{\lambda}$ are locally defined lifts of $\gamma$ and $\lambda_{L}$. Since the image of $\gamma$ is contained in a connected component of $U_{L}$, both $\mu(\widetilde{\lambda}(p), \widetilde{\gamma}(p))$ and $\mu\left(\widetilde{\gamma}(p), \lambda_{\infty}(p)\right)$ are constant on $\pi(U)$. The difference between the shifts can be calculated as

$$
\begin{aligned}
d(p)-d(q) & =\frac{1}{2}\left(\tau\left(\lambda_{\infty}\left(p^{\prime}\right), \lambda_{\widehat{L}}\left(p^{\prime}\right), \gamma\left(p^{\prime}\right)\right)-\tau\left(\lambda_{\infty}\left(q^{\prime}\right), \lambda_{\widehat{L}}\left(q^{\prime}\right), \gamma\left(q^{\prime}\right)\right)\right) \\
& =\mu\left(\lambda_{\infty}(p), \widetilde{\lambda}(p)\right)-\mu\left(\lambda_{\infty}(q), \widetilde{\lambda}(q)\right)
\end{aligned}
$$

(see Gui12, Section 8]). Hence the function $d(p)-\mu\left(\lambda_{\infty}(p), \widetilde{\lambda}(p)\right)$ is constant on $\pi(U)$ with value in $\frac{1}{2} \mathbb{Z}$. Moreover, since $\mu(\widetilde{\lambda}(p), \gamma(p)) \equiv \mu\left(\gamma(p), \lambda_{\infty}(p)\right) \equiv \frac{1}{2} \operatorname{dim} M \bmod \mathbb{Z}$, we have

$$
d(p)-\mu\left(\lambda_{\infty}(p), \widetilde{\lambda}(p)\right) \equiv \frac{1}{2} \operatorname{dim}(M \times \mathbb{R})=\frac{1}{2}(\operatorname{dim} M+1) \quad \bmod \mathbb{Z} .
$$

By Proposition C.3(v), $\tilde{\lambda}$ can be uniquely chosen so that (C.11) holds on $\pi(U)$. Such $\tilde{\lambda}$ can be glued together on the whole of $L$ and becomes a grading of $L$. 
Next we consider the degree of $\mathcal{H o m}^{\star}\left(F_{2}, F_{1}\right)$. Let $L_{1}$ and $L_{2}$ be compact exact Lagrangian submanifolds of $T^{*} M$ intersecting cleanly. For $i=1,2$, take a primitive $f_{i}: L_{i} \rightarrow \mathbb{R}$ of the Liouville 1 -form and denote by $\widehat{L}_{i}$ the conification of $L_{i}$ with respect to $f_{i}$. Let $F_{i} \in \mathbf{D}^{\mathrm{b}}(M \times \mathbb{R})$ be a simple sheaf quantization of $\widehat{L_{i}}$. We also denote by $d_{i}: L_{i} \rightarrow \frac{1}{2} \mathbb{Z}$ the function which assigns the shift of $F_{i}$. Then by Theorem 4.14, the degree associated with a component $C$ of $L_{1} \cap L_{2}$ in $\mathcal{H o m}^{\star}\left(F_{2}, F_{1}\right)$ is given by

$$
d_{2}(p)-d_{1}(p)+\frac{1}{2}(\operatorname{dim} M-\operatorname{dim} C)-\frac{1}{2} \tau\left(T_{p} L_{2}, T_{p} L_{1}, \lambda_{\infty}(p)\right)
$$

for any $p \in C$. Thus, combining Proposition C.5 with (C.10) and (C.15), we obtain the following theorem.

Theorem C.6. For $i=1,2$, let $\widetilde{\lambda_{i}}: L_{i} \rightarrow \widetilde{\mathcal{L}}_{T^{*} M}$ be the grading of $L_{i}$ given in Proposition C.5. Then the degree associated with a component $C$ of $L_{1} \cap L_{2}$ in $\mathcal{H o m}^{\star}\left(F_{2}, F_{1}\right)$ is equal to $\operatorname{gr}\left(L_{2}, L_{1} ; C\right)$.

\section{References}

[Abo12] M. Abouzaid, Nearby Lagrangians with vanishing Maslov class are homotopy equivalent, Invent. Math., 189 (2012), no. 2, 251-313.

[dG09] M. de Gosson, On the usefulness of an index due to Leray for studying the intersections of Lagrangian and symplectic paths, J. Math. Pures Appl. (9), 91 (2009), no. 6, 598-613.

[FOOO09a] K. Fukaya, Y.-G. Oh, H. Ohta, and K. Ono, Lagrangian intersection Floer theory: anomaly and obstruction. Part I, Vol. 46 of AMS/IP Studies in Advanced Mathematics, American Mathematical Society, Providence, RI; International Press, Somerville, MA, 2009.

[FOOO09b] K. Fukaya, Y.-G. Oh, H. Ohta, and K. Ono, Lagrangian intersection Floer theory: anomaly and obstruction. Part II, Vol. 46 of AMS/IP Studies in Advanced Mathematics, American Mathematical Society, Providence, RI; International Press, Somerville, MA, 2009.

[Fra04] U. Frauenfelder, The Arnold-Givental conjecture and moment Floer homology, Int. Math. Res. Not., (2004), no. 42, 2179-2269.

[FSS08] K. Fukaya, P. Seidel, and I. Smith, Exact Lagrangian submanifolds in simplyconnected cotangent bundles, Invent. Math., 172 (2008), no. 1, 1-27.

[FSS09] K. Fukaya, P. Seidel, and I. Smith, The symplectic geometry of cotangent bundles from a categorical viewpoint, In Homological mirror symmetry, Vol. 757 of Lecture Notes in Phys. 1-26, Springer, Berlin, 2009.

[GKS12] S. Guillermou, M. Kashiwara, and P. Schapira, Sheaf quantization of Hamiltonian isotopies and applications to nondisplaceability problems, Duke Math. J., 161 (2012), no. 2, 201-245.

[GS14] S. Guillermou and P. Schapira, Microlocal theory of sheaves and Tamarkin's non displaceability theorem, In Homological mirror symmetry and tropical geometry, Vol. 15 of Lect. Notes Unione Mat. Ital. 43-85, Springer, Cham, 2014. 
[Gui12] S. Guillermou, Quantization of conic Lagrangian submanifolds of cotangent bundles, arXiv preprint, arXiv:1212.5818v2, (2012).

[Gui16] S. Guillermou, Quantization of exact Lagrangian submanifolds in a cotangent bundle, lecture notes available at the author's webpage, https://www-fourier.ujf-grenoble.fr/ ${ }^{\sim}$ guillerm/, (2016).

[KO01] R. Kasturirangan and Y.-G. Oh, Floer homology of open subsets and a relative version of Arnold's conjecture, Math. Z., 236 (2001), no. 1, 151-189.

[Kra13] T. Kragh, Parametrized ring-spectra and the nearby Lagrangian conjecture, Geom. Topol., 17 (2013), no. 2, 639-731. With an appendix by Mohammed Abouzaid.

[KS90] M. Kashiwara and P. Schapira, Sheaves on manifolds, Vol. 292 of Grundlehren der Mathematischen Wissenschaften, Springer-Verlag, Berlin, 1990.

[Ler81] J. Leray, Lagrangian analysis and quantum mechanics, MIT Press, Cambridge, Mass.-London, 1981, A mathematical structure related to asymptotic expansions and the Maslov index, Translated from the French by Carolyn Schroeder.

[Nad09] D. Nadler, Microlocal branes are constructible sheaves, Selecta Math. (N.S.), 15 (2009), no. 4, 563-619.

[NZ09] D. Nadler and E. Zaslow, Constructible sheaves and the Fukaya category, $J$. Amer. Math. Soc., 22 (2009), no. 1, 233-286.

[Poź99] M. Poźniak, Floer homology, Novikov rings and clean intersections, In Northern California Symplectic Geometry Seminar, Vol. 196 of Amer. Math. Soc. Transl. Ser. 2 119-181, Amer. Math. Soc., Providence, RI, 1999.

[RS93] J. Robbin and D. Salamon, The Maslov index for paths, Topology, 32 (1993), no. $4,827-844$.

[Sch16] F. Schmäschke, Floer homology of Lagrangians in clean intersection, arXiv preprint, arXiv:1606.05327, (2016).

[Sei00] P. Seidel, Graded Lagrangian submanifolds, Bull. Soc. Math. France, 128 (2000), no. 1, 103-149.

[ST92] P. Schapira and N. Tose, Morse inequalities for R-constructible sheaves, Adv. Math., 93 (1992), no. 1, 1-8.

[Tam18] D. Tamarkin, Microlocal condition for non-displaceability, in Algebraic and Analytic Microlocal Analysis, Springer Proceedings in Mathematics \& Statistics 269, Springer, Cham, 2018, 99-223.

Yuichi Ike

Fujitsu Laboratories Ltd., 4-1-1 Kamikodanaka, Nakahara-ku, Kawasaki, Kanagawa, 2118588, Japan

E-mail address: yuichi.ike.1990@gmail.com, ike.yuichi@fujitsu.com 